Revue des patrimoines

\title{
Des chevaux réels et un cheval idéal : naturalisme et idéalisation des chevaux des portraits équestres italiens des Trecento et Quattrocento
}

\section{Armelle Fémelat}

\section{(2) OpenEdition}

\section{Journals}

Édition électronique

URL : http://journals.openedition.org/insitu/12040

DOI : 10.4000/insitu. 12040

ISSN : 1630-7305

Éditeur

Ministère de la culture

Référence électronique

Armelle Fémelat, " Des chevaux réels et un cheval idéal : naturalisme et idéalisation des chevaux des portraits équestres italiens des Trecento et Quattrocento », In Situ [En ligne], 27 | 2015, mis en ligne le 02 novembre 2015, consulté le 30 avril 2019. URL : http://journals.openedition.org/insitu/12040 ; DOI : 10.4000/insitu. 12040

Ce document a été généré automatiquement le 30 avril 2019.

\section{(c) (i) ()}

In Situ Revues des patrimoines est mis à disposition selon les termes de la licence Creative Commons Attribution - Pas d'Utilisation Commerciale - Pas de Modification 4.0 International. 


\title{
Des chevaux réels et un cheval idéal: naturalisme et idéalisation des chevaux des portraits équestres italiens des Trecento et Quattrocento
}

\author{
Armelle Fémelat
}

Dans une thèse intitulée Le Portrait équestre italien de la fin du Moyen Age au début de la Renaissance soutenue en $2010^{1}$, je démontrai l'outil de communication politique que constitua ce type de portrait officiel à forte valence martiale au bénéfice de certains détenteurs de commandements militaires. Instrument d'authentification du pouvoir utilisé à des fins de légitimation, voire de propagande, les effigies équestres des Trecento et Quattrocento furent partie prenante de la réalité de l'art et de la représentation comme exercice du pouvoir. Je remarquai en outre que leurs montures manifestent une double tendance, a priori paradoxale, au naturalisme et à l'idéalisation. Ces représentations équines gagnent en effet à être analysées du point de vue de leur rapport à la réalité et à l'idéalité équines, via les pratiques artistiques innovantes de l'époque. Naturalisme versus idéalisation, telle est bien la nature d'un des enjeux artistiques qui s'est alors joué dans la péninsule italienne et qu'illustrent pleinement les chevaux des portraits équestres italiens des $\mathrm{XIV}^{\mathrm{e}}$ et $\mathrm{XV}^{\mathrm{e}}$ siècles.

\section{Des chevaux réels - une tendance au naturalisme}

Variable selon les époques, les lieux, les artistes et au cas par cas, la tendance au naturalisme décelable dans les représentations prend différentes formes. Perceptible tant dans les portraits équestres que dans diverses études et travaux préparatoires, cette tendance croissante au naturalisme fait écho à l'évolution des conceptions et des pratiques artistiques alors à l'œuvre. Une telle évolution dans l'art de la représentation équine induisait notamment un regard inédit sur les chevaux qui passait par l'observation 
et la figuration des équidés d'après nature, comme l'attestent les études morphologiques de Pisanello ainsi que les études anatomiques et les recherches dans la représentation du mouvement de Léonard de Vinci.

\section{Le naturalisme des portraits équestres des Trecento et Quattrocento}

3 Les portraits équestres des $\mathrm{XIV}^{\mathrm{e}}$ et $\mathrm{XV}^{\mathrm{e}}$ siècles donnent à voir des manifestations complémentaires d'une volonté de représentation de la réalité équine contemporaine, que ce soit par le biais de modèles caractérisés et de types de chevaux identifiables, ou encore de détails choisis de morphologie ou de physiologie équines, incluant les traces de certaines pratiques hippologiques et hippiatriques.

\section{Des modèles caractérisés et des types de chevaux identifiables}

4 Aux Trecento et Quattrocento, le cheval occupait une place de premier plan dans la vie et dans la société italiennes. De plus, son usage se diversifiait. Cette diversification a été progressive, au gré de la circulation toujours croissante des chevaux dans la Péninsule, en Occident et par delà, au Proche-Orient et en Afrique du Nord notamment. Recherchée par l'homme, la spécialisation de l'animal répondait à la spécialisation de l'utilisation qu'il en faisait et conduisit à l'essor de razze - dans le sens que revêtait cette terminologie dans la seconde moitié du Quattrocento et qui constitue le point de départ sémiologique de l'acception actuelle du terme « race ». Le concept de race équine émergea en effet à la fin $\mathrm{du} \mathrm{Xv} \mathrm{e}^{\mathrm{e}}$ siècle, en écho aux pratiques inédites mises en œuvre dans les élevages des Aragon à Naples, des Gonzague à Mantoue et des Este à Ferrare. Reflets de cette spécialisation équine, plusieurs effigies équestres des Trecento et Quattrocento donnent à voir des modèles de chevaux aisément identifiables, de type napolitain ou oriental.

\section{Les chevaux de type napolitain}


Figure 1

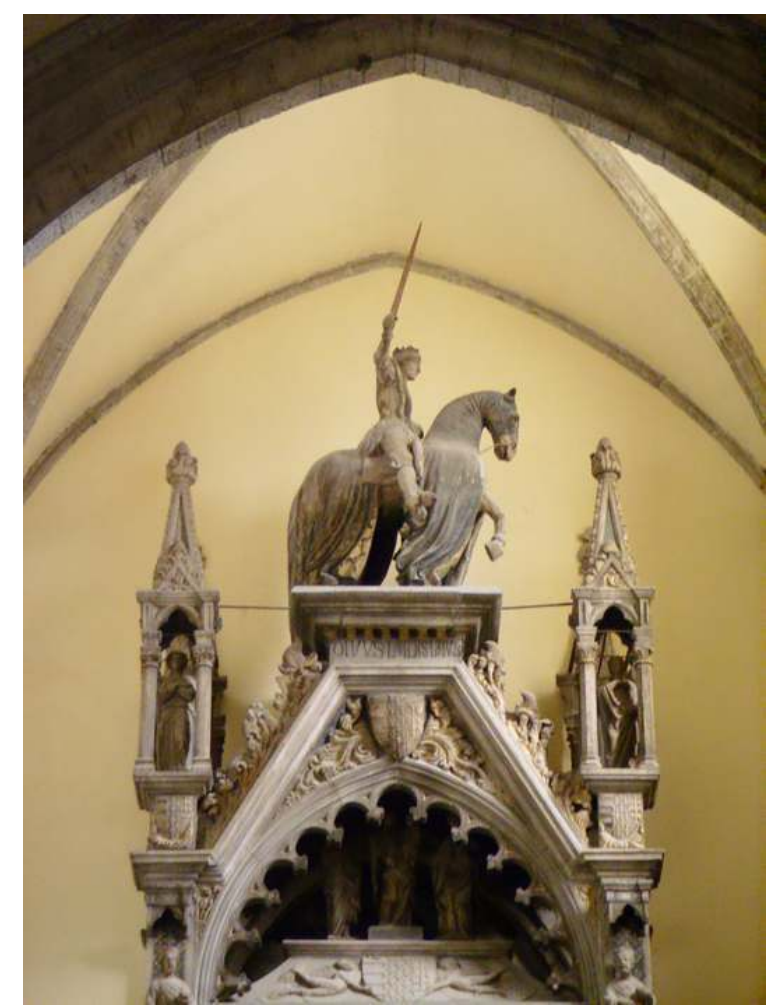

Anonyme, statue équestre sommitale du Monument funéraire de Ladislas d'Anjou-Duras, ronde-bosse, marbre. Naples, San Giovanni a Carbonara, chœur, in situ, 1428 (daté)-ca 1432.

Phot. Fémelat, Armelle. (c) Armelle Fémelat, 2007.

$5 \quad$ Nettement plus haut et plus robuste que le type oriental, avec le poitrail et la croupe bien éclatés, le cheval de type napolitain se reconnaît à son encolure en col de cygne, longue, haute et rouée - c'est-à-dire convexe au niveau de la jonction avec la tête - ainsi qu'à sa tête, relativement longue, avec un chanfrein convexe. Il apparaît dans trois portraits équestres de rois de Naples du Quattrocento : la statue équestre sommitale du Monument funéraire de Ladislas d'Anjou-Duras à San Giovanni a Carbonara de Naples ${ }^{2}$ (fig. 1) et deux enluminures à l'effigie de Ferrante $\mathrm{I}^{\mathrm{er}} \mathrm{d}^{\prime}$ Aragon $^{3}$ (fig. 2). Cela étant, déjà au milieu du XIV ${ }^{\mathrm{e}}$ siècle, les chevaux de combat des statues de Guidoriccio da Fogliano ${ }^{4}$, de Cangrande ${ }^{5}$ (fig. 3) et de Mastino II Della Scala ${ }^{6}$ arboraient une même longue encolure de cygne surmontée d'une tête au profil légèrement convexe - sans qu'ils soient pour autant assimilables au type napolitain eu égard aux autres critères distinctifs du type. 


\section{Figure 2}

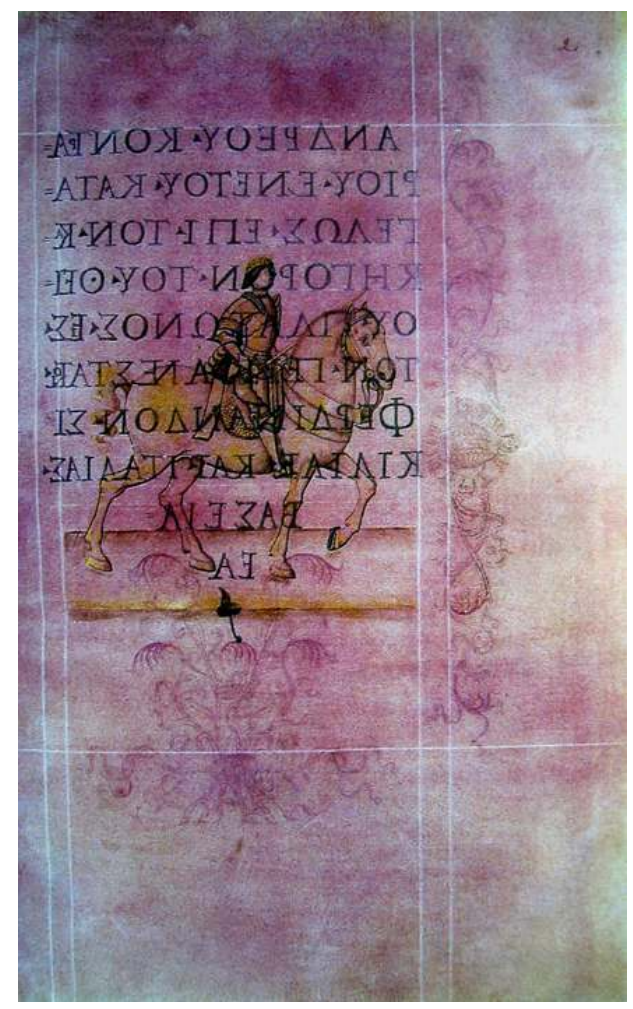

Cola Rapicano (documenté), Portrait équestre de Ferrante ler d'Aragon, enluminure. Paris, Bibliothèque nationale de France, département des Manuscrits occidentaux, Ms Lat. 12947, folio 2, 1471 (daté).

Phot. Fémelat, Armelle. (c) Armelle Fémelat, 2008. 


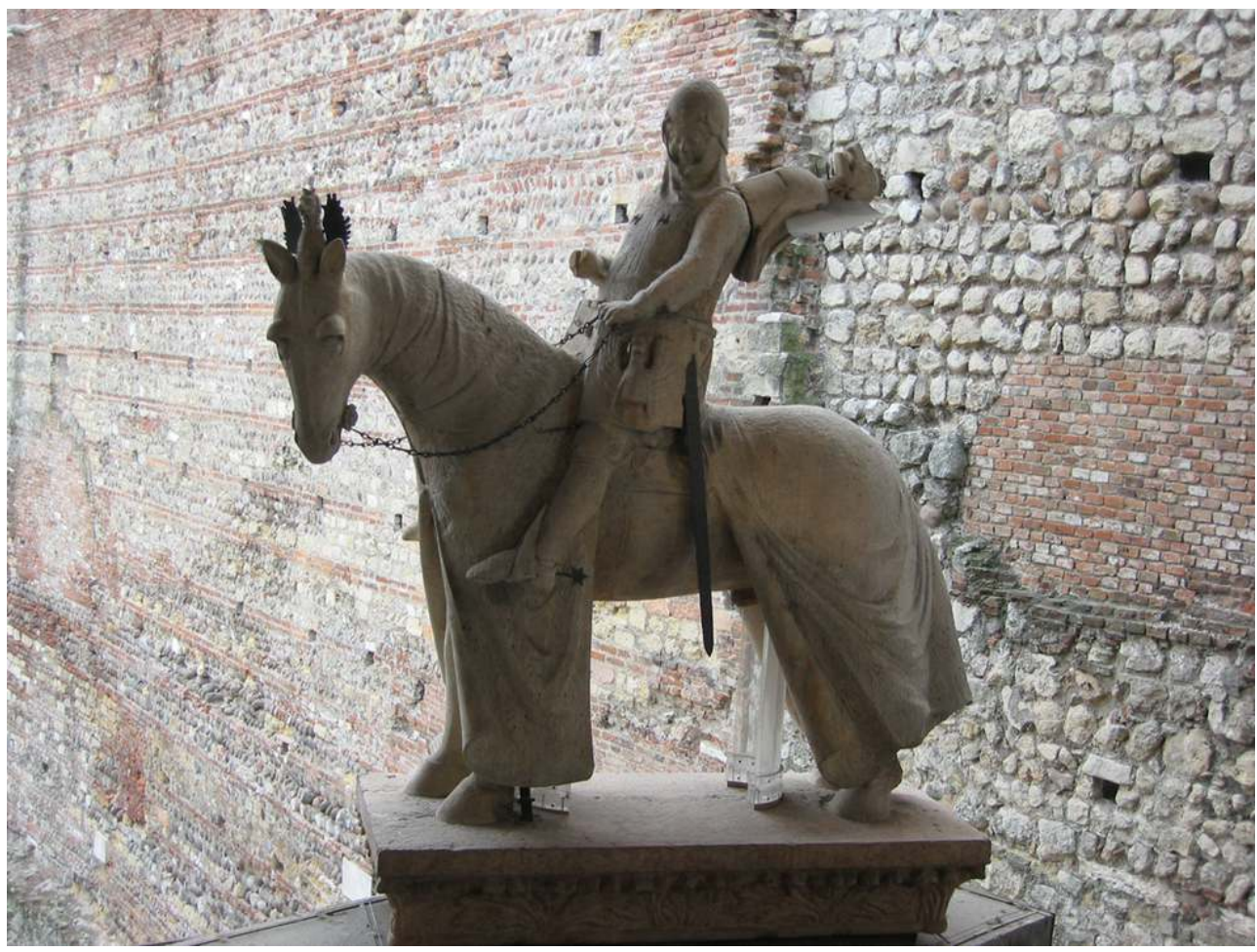

Anonyme, statue équestre sommitale du Monument funéraire de Cangrande Della Scala, ronde-bosse, pierre. Vérone, Museo di Castelvecchio, provenant du cimetière Scaliger attenant à Santa Maria Antica de Vérone, ca 1340-1345.

Phot. Fémelat, Armelle. ㄷ Armelle Fémelat, 2003.

6 Amplement utilisé comme monture de guerre à la fin du Moyen Âge, le cheval napolitain bénéficiait alors d'une réputation sans égale et il fit l'objet d'un commerce international considérable. Puis, au XVI ${ }^{\mathrm{e}}$ siècle, il connut un second âge d'or, au moment de l'apparition et du développement des académies d'art équestre. Apprécié au fil des siècles, tant pour ses qualités esthétiques que pour ses aptitudes naturelles, et bien que disparu depuis plus d'un siècle, il continue de susciter admiration et passion - au point que le Napolitain Giuseppe Maresca lui consacre des recherches historiques depuis les années 1970 et développe une politique d'élevage dans le but d'en « restaurer » la race ${ }^{7}$.

\section{Les chevaux de type oriental}

7 D'autres effigies équestres représentent les montures de type oriental de deux souverains orientaux qui se sont succédé, un empereur romain d'Orient et un empereur ottoman. 
Figure 4

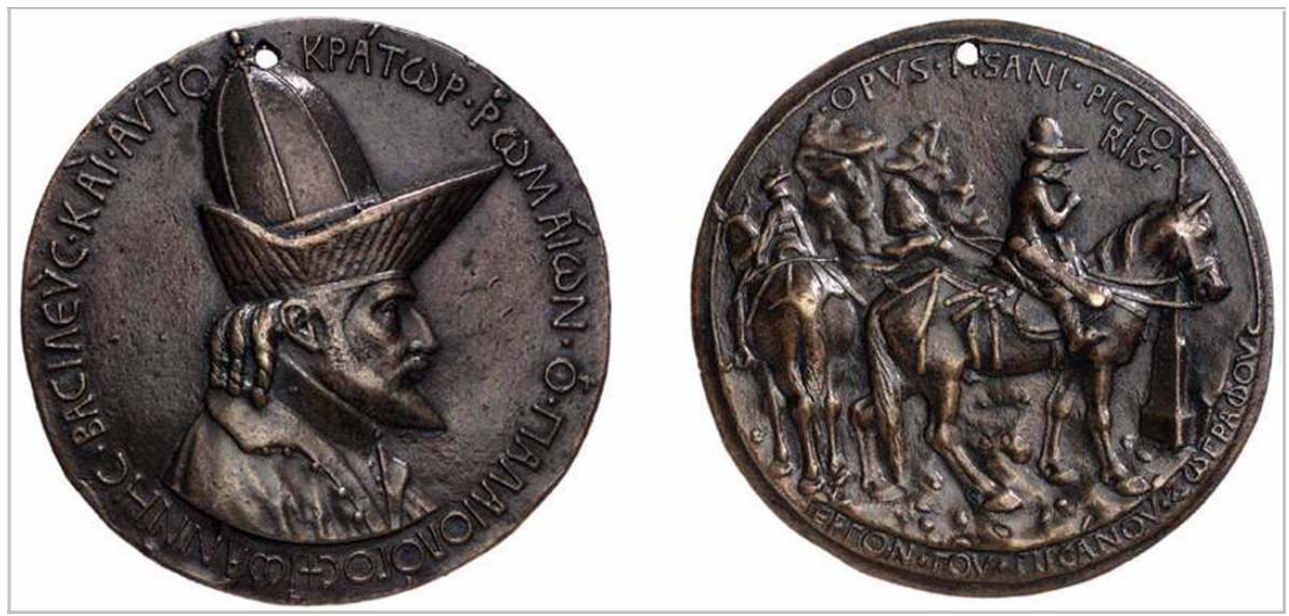

Pisanello (signé), Médaille à l'effigie de Jean VIII Paléologue, bas-relief, bronze. Paris, Bibliothèque nationale de France, département des Monnaies, Médailles et Antiques, AV 17, ca 1438-1439.

Phot. Fémelat, Armelle. (c) Armelle Fémelat, 2008.

8 Le modèle très particulier du cheval de Jean VIII Paléologue sur le revers de la médaille de Pisanello $^{8}$ (fig. 4) s'avère de type oriental, et plus précisément danubien. Caractérisé par sa très petite taille, sa silhouette gracile, son corps long, sa grosse tête et ses grandes oreilles, il se distingue très nettement des nombreux chevaux habituellement dessinés par l'artiste - massifs, avec de petites oreilles, la croupe et l'encolure arrondies. Ce même cheval danubien apparaît également dans deux études préparatoires à la médaille ${ }^{9}$ ainsi que dans deux autres études du maître : une étude de tête ${ }^{10}$ et une étude de corps, vu de face et de $\operatorname{dos}^{11}$ (fig. 5). Les circonstances historiques qui ont amené Pisanello à représenter un tel cheval - monture attestée de l'empereur byzantin durant son séjour florentin à l'occasion du concile des Deux Églises - sont aujourd'hui bien connues ${ }^{12}$. Dans ses Mémoires, Syropoulos affirme en effet que l'empereur et son frère, le despote Demetrios, achetèrent des montures de chasse à Nicolas Gydeles ${ }^{13}$, lequel se rendit au concile avec la délégation russe menée par le métropolite de Kiev, Isidore, en août 1438. Dans ses études, Pisanello ne s'est pas focalisé sur le seul cheval impérial, il a en outre minutieusement détaillé les costumes et les accessoires des membres de la délégation orientale présente au concile. Esquissées à la manière d'un reportage pris sur le vif, de telles études traduisent la curiosité suscitée par cette délégation et l'indéniable attrait que l'Orient exerçait alors sur les artistes italiens. 


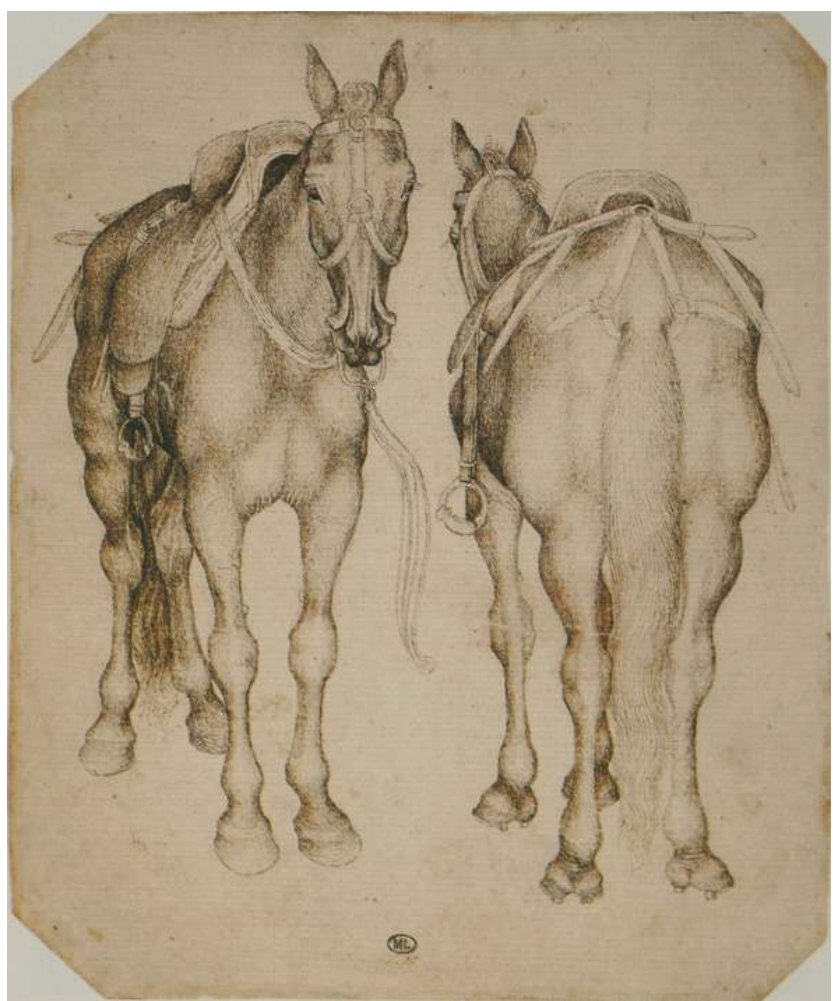

Attribuée à Pisanello, Étude d'un cheval oriental aux naseaux fendus vu de trois-quarts face et de troisquarts dos, plume et encre brune, lavis gris et brun, rehauts de blanc. Paris, musée du Louvre, département des Arts graphiques, Inventaire 2468, ca 1438.

Phot. Fémelat, Armelle. (c) Armelle Fémelat, 2005.

Le Portrait équestre de Mehmet II qui orne le revers de la médaille conçue par Constanzo da Ferrara durant l'hiver $1480-1481^{14}$ montre un second type de cheval oriental. De petite taille, les formes arrondies, les membres délicats et les attaches fines, la monture du sultan se distingue surtout par la hauteur du port de sa petite tête - aux yeux écartés et au chanfrein concave - ainsi que par l'attache, haute et horizontale, de sa queue. Un tel modèle se révèle morphologiquement proche de l'actuel type du pur-sang arabe caractérisé par sa finesse, sa taille modeste, la hauteur de ses ports de tête et de queue, ainsi que la forme concave de son chanfrein -, traditionnellement appelé oriental ou arabe, dont l'existence en tant que race officielle ne remonte qu'au $\mathrm{xIx}^{\mathrm{e}}$ siècle mais dont la réalité historique est attestée dès le haut Moyen Âge ${ }^{15}$. De surcroît, les chevaux de plusieurs statues équestres de la seconde moitié $\mathrm{du} \mathrm{Xv}^{\mathrm{e}}$ siècle - dont celles du Gattamelata par Donatello ${ }^{16}$ (fig. 6), d'Antonio Rido ${ }^{17}$, de Giordano Orsini ${ }^{18}$ et de Bartolomeo Colleoni par Andrea del Verrochio ${ }^{19}$ - arborent la même petite tête dite camuse - caractérisée par la ligne concave reliant le front au bout du nez - à l'exclusion des autres caractéristiques morphologiques du type arabe. 


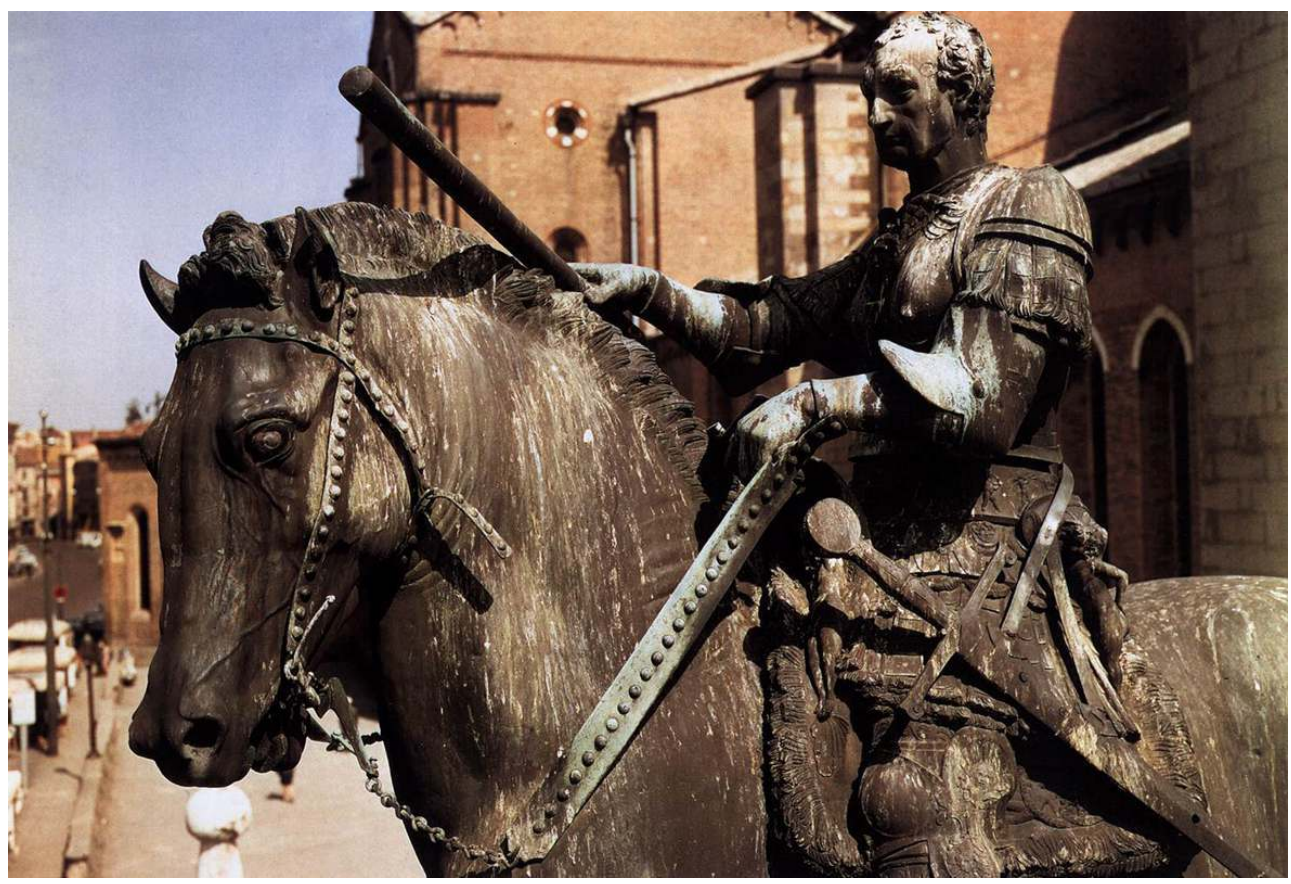

Donatello (signé), tête et encolure du cheval de la Statue équestre du Gattamelata, ronde-bosse, bronze. Padoue, place du Santo, in situ, 1447-1453 (documenté).

Phot. Fémelat, Armelle. (C) Armelle Fémelat, 2003.

Nonobstant ces quelques types équins identifiables, la tendance au naturalisme des chevaux des portraits équestres ne se limite pas au seul modèle de la monture, elle concerne également certains détails choisis.

\section{Des détails de morphologie et de physiologie équines}

11 En préambule à l'étude des détails de morphologie et de physiologie équines qui ont été observés et figurés avec un soin particulier - les attributs sexuels ainsi que les veines affleurant sous le pelage et les plis de la peau, les naseaux dilatés, la bouche ouverte et la position des oreilles -, il est utile de préciser que le rendu de ces détails est très inégal selon les effigies considérées, tant au niveau de la précision de la représentation que du rendu des détails ou des matières.

\section{Organes génitaux mâles}

Bien que parfois disgracieux et peu réalistes au regard de la posture et de la physionomie de l'animal, les attributs sexuels mâles - bourse et/ou fourreaux - n'en sont pas moins une constante des portraits équestres italiens des $\mathrm{XIV}^{\mathrm{e}}$ et $\mathrm{XV}^{\mathrm{e}}$ siècles. Plus encore, tout semble avoir été fait pour accroître la visibilité d'un tel détail anatomique, ô combien symbolique! Cependant, faute de sources, il demeure impossible de savoir si cette sexuation ostensible est venue répondre à une demande explicite des commanditaires, si elle a été le fruit d'une initiative des artistes, ou si elle résultait d'une convention iconographique. Comme l'atteste nombre de représentations équestres occidentales de l'Antiquité à nos jours, le cheval entier est un poncif des représentations officielles d'autant plus lorsque ces représentations se doublent d'une dimension martiale, ce qui 
est précisément le cas des portraits équestres. Hautement symbolique, ce détail morphologique exprime en effet la virilité, la combativité et le courage, autant de qualités qui, par extension, contribuent à caractériser le cavalier. Les organes génitaux mâles comptent ainsi parmi les « signes du pouvoir » du portrait équestre, la morphologie et les qualités équines étant partie prenante de l'instrument de communication politique qu'il constitue, y compris dans ses implications artistiques ${ }^{20}$.

Les dessins et les esquisses de chevaux réalisés d'après nature par Pisanello et Léonard de Vinci dévoilent souvent, et de manière significative, cette partie anatomique ${ }^{21}$ (fig. 7), quand elle ne constitue pas l'unique objet de l'étude ${ }^{22}$. De surcroît, aux XIV ${ }^{\mathrm{e}}$ et $\mathrm{XV}^{\mathrm{e}}$ siècles, l'attention portée à l'identification sexuelle - mâle - de la monture ne se limita pas aux images, elle fut tout aussi récurrente dans les écrits, de toute nature: documents comptables, traités scientifiques et techniques, textes littéraires, etc. De ce point de vue, les textes médiévaux, dans lesquels destrier rime généralement avec entier ${ }^{23}$, prolongèrent l'héritage antique. En effet, depuis Xénophon, le cheval idéal pour combattre et pour pratiquer une équitation digne de ce nom était inéluctablement entier. Au Moyen Âge, à la Renaissance et longtemps encore, l'idée perdura en Occident que tout cheval de qualité, destiné à la selle des élites - au combat et à la parade - ne devait pas être soumis à la castration. Opération qui aurait eu pour conséquence de priver l'animal de sa fougue et de son courage, autant de qualités essentielles, signifiantes et qualifiantes pour son cavalier. Par ailleurs, à en croire les sources, le prestige et le prix du cheval entier se fondaient en partie sur son aptitude de reproducteur ${ }^{24}$.

Figure 7

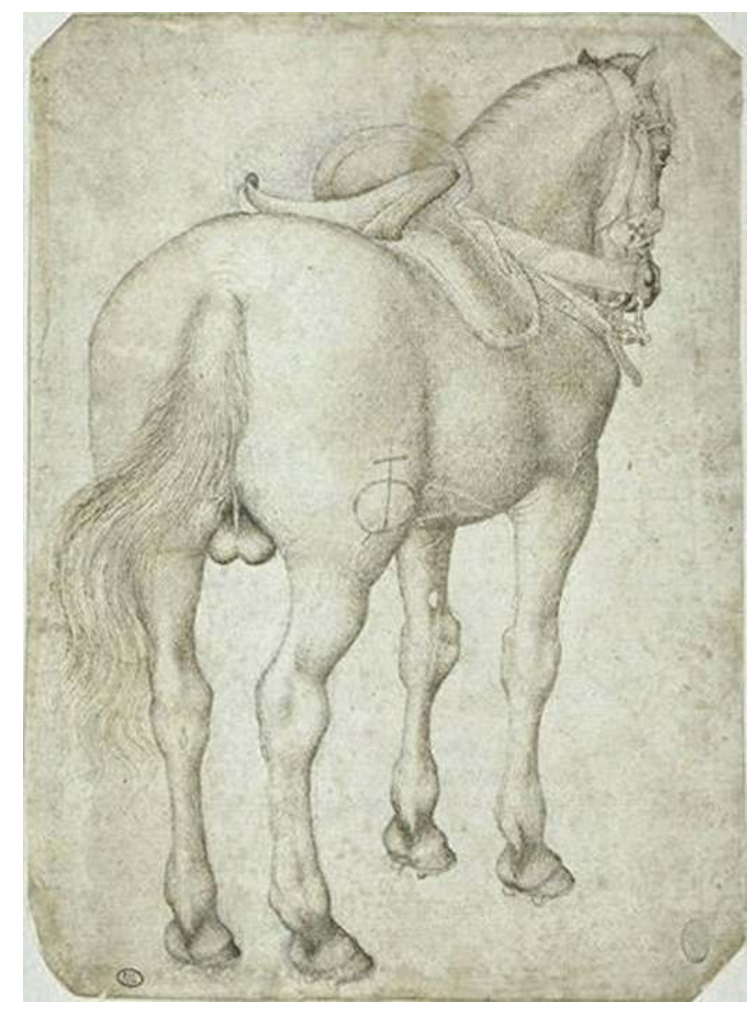

Attribuée à Pisanello, Étude d'un cheval harnaché de trois-quarts dos, plume et encre brune, tracé préparatoire à la pierre noire sur papier. Paris, musée du Louvre, département des Arts graphiques, Inventaire 2378, ca.1435.

Phot. Fémelat, Armelle. (C) Armelle Fémelat, 2005 
Bien que ne reflétant pas la réalité historique, l'omniprésence des entiers dans les représentations a néanmoins pour corollaire un mépris affiché à l'encontre des juments et des hongres (mâles castrés). Mais là encore, il existe un vrai décalage entre le contenu des représentations, textuelles en particulier, et la réalité telle qu'elle se dessine à travers les sources. En effet, si certains auteurs exprimèrent des réserves quant à l'efficacité des juments en tant que montures, en particulier sur le champ de bataille, ils n'en reconnurent pas moins leur valeur, leurs mérites et leurs qualités morphologiques, également en tant que reproductrices. Et dans les faits, les juments prirent pleinement part aux combats et aux diverses activités guerrières. Les documents d'archives établissent clairement qu'elles furent fréquemment associées aux usages les plus prestigieux - et les plus symboliques - de la guerre, des jeux chevaleresques et des parades $^{25}$, bien loin de l'idée reçue selon laquelle elles ne furent que des montures de femmes, et d'ecclésiastiques dans le cas des haquenées.

\section{Autres détails anatomiques remarquables des effigies équestres, la bouche et les naseaux}

\section{Bouches ouvertes}

Dans leur grande majorité, les chevaux des effigies équestres sont figurés la bouche ouverte. Visuellement inattendu, ce détail s'avère néanmoins réaliste du point de vue éthologique, puisque les chevaux montés jouent facilement avec leur mors: ils ouvrent légèrement la bouche, en signe de décontraction, de concentration et d'application dans l'exécution des ordres transmis. De surcroit, généralement la bouche est représentée suffisamment ouverte pour laisser voir les dents de l'animal. Or, depuis l'Antiquité, elles constituent un détail iconographique couramment utilisé pour évoquer l'agressivité et la combativité équines. De fait, elles sont une «arme » corporelle de première importance dans les techniques de combat naturelles de l'animal. Et figurer une monture bouche ouverte, toutes dents dehors, lui confère une indéniable valence martiale.

Ainsi, sur les deux revers de médaille conçus par Gianfrancesco Enzola en 1473 et en 1475, le cheval cabré de Constanzo Sforza s'élance au galop, dents sorties et oreilles couchées ${ }^{26}$. Détails que l'on retrouve sur le relief d'Annibale Bentivoglio réalisé à Bologne un quart de siècle auparavant ${ }^{27}$ (fig. 8). Le fait de figurer un cheval avec les oreilles en arrière et les dents visibles évoque les techniques d'attaque et de défense de l'animal se cabrant pour frapper son adversaire des antérieurs et le mordre. Situation que Léonard de Vinci a précisément reproduite dans plusieurs études préparatoires des années 1503-1504 pour la Bataille d'Anghiari ${ }^{28}$. L'une d'elles en particulier montre un cheval cabré secouant la tête, toutes dents dehors. Fait significatif, l'artiste a reproduit à cinq reprises le détail de la bouche ouverte laissant voir dents et mâchoires ${ }^{29}$. De même, plusieurs chevaux antiques de bronze du Musée archéologique de Naples exhibent une bouche grande ouverte, avec des dents bien visibles, des veines gonflées et des naseaux dilatés - véritable manifeste de leur agressivitée ${ }^{30}$. C'est le cas notamment du Protomé Médicis, antique ${ }^{31}$ dont le Protomé Carafa de Donatello (ca 1455) ${ }^{32}$ reprend la formule, sans doute en lien avec la commande d'une statue équestre du roi de Naples, Alphonse $V$ d'Aragon, finalement jamais réalisée. 
Figure 8

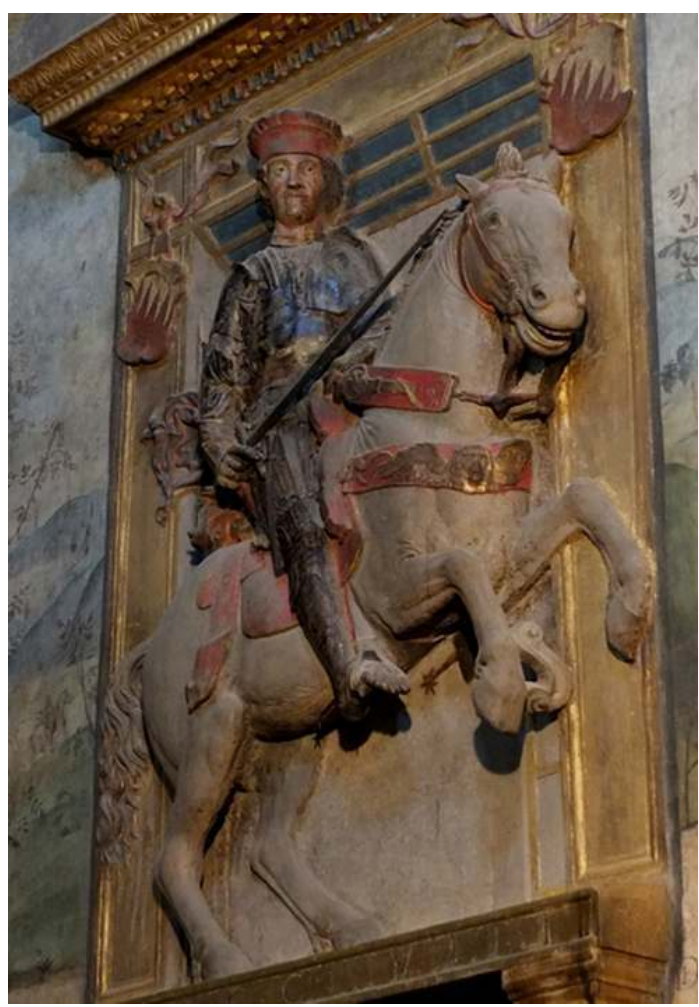

Anonyme, Portrait équestre funéraire d'Annibale Bentivoglio, haut-relief, terre cuite polychrome. Bologne, San Giacomo Maggiore, chapelle Bentivoglio, in situ, 1458.

Phot. Fémelat, Armelle. (c) Armelle Fémelat, 2005.

\section{Naseaux dilatés}

Les naseaux dilatés comptent parmi les détails physiologiques équins souvent reproduits par les artistes de la Renaissance, en particulier dans les effigies équestres. Véritables organes d'expression physionomique, dont la dilatation - resserrement, crispation ou flaccidité - traduit l'état physique de l'animal ainsi que sa vigueur. Le fait de représenter un cheval avec les naseaux ostensiblement dilatés permet d'indiquer qu'il est fringant, en train de réaliser un effort physique intense.

Avec la Statue équestre de Bernabò Visconti, Bonino da Campione proposa dès 1355-136033, un véritable "portrait de museau $»^{34}$ au traitement naturaliste, à la fois précis et illusionniste, totalement inédit pour l'époque ${ }^{35}$. Ses naseaux dilatés semblent frémir, tandis que le réseau des plis de la peau, très fine, sous laquelle affleurent les veines, s'articule autour des yeux et de la bouche d'une façon remarquable. De plus, le sculpteur s'est appliqué à figurer l'épiderme de l'animal et le mouvement des poils grâce à un traitement subtil de la surface sculptée, à l'origine peinte dans un esprit naturaliste (al naturale).

En outre, plusieurs dessins de Léonard ${ }^{36}$, dont certaines études préparatoires pour les projets Sforza et Trivulzio ${ }^{37}$, montrent des chevaux aux naseaux hypertrophiés. Ce détail physiologique concourt au but commun de nombreux écrits et dessins léonardiens d'évoquer la vie et le mouvement. L'artiste analysa précisément le système respiratoire équin. Dans une note, il explique : 
La nature a fait dans le nez deux trous dont chacun a la moitié de la largeur du tuyau par où s'exhale le souffle des poumons ; même si ces trous n'existaient pas, la bouche aurait suffi à l'abondance de souffle. Si tu me demandes pourquoi la nature a ainsi pourvu les animaux de naseaux, alors qu'ils leur suffiraient de respirer par la bouche, je te répondrai que les naseaux servent quand la bouche est occupée à mastiquer des éléments ${ }^{38}$.

Dans le Traité sur le cheval qu'il écrivit vers 1445, Leon Battista Alberti classa les «narines bien gonflées et largement ouvertes " parmi les critères de sélection d'un bon cheval reproducteur ${ }^{39}$. Ayant précisé plus avant que «les chevaux appelés à avoir le meilleur caractère » ont les oreilles mobiles « insatiables, méfiantes, palpitantes » ${ }^{40}$.

\section{Oreilles mobiles et expressives}

21 Les oreilles constituent un autre détail de la tête du cheval évocateur de son comportement. Leur direction et leur attitude - fixe ou mobile - expriment l'état physique et psychologique, le caractère ainsi que les réactions sensibles de l'animal. Pointées en avant, elles signalent son attention et sa confiance, couchées en arrière, elles expriment sa méchanceté ou sa contrariété tandis que tournées vers l'extérieur, elles dénotent une certaine curiosité voire de l'anxiété.

Dans la majorité des portraits équestres, les chevaux sont figurés avec les oreilles en avant, un nombre non négligeable avec les oreilles tournées sur le côté, et seulement quelques-uns avec les oreilles couchées en arrière - ces derniers arborant aussi des naseaux pincés, autre indice de la méfiance de l'animal. Conçue par Verrocchio dans la décennie 1480, la monture de Bartolomeo Colleoni ${ }^{41}$ (fig. 9) a l'oreille gauche tournée vers l'arrière et la droite pointée en avant. Ce moyen figuratif suggère le mouvement des oreilles qui accompagne la marche attentive d'une monture sollicitée; il contribue à incarner l'effigie du condottiere et à faire de son cheval un auxiliaire à l'écoute. 
Figure 9

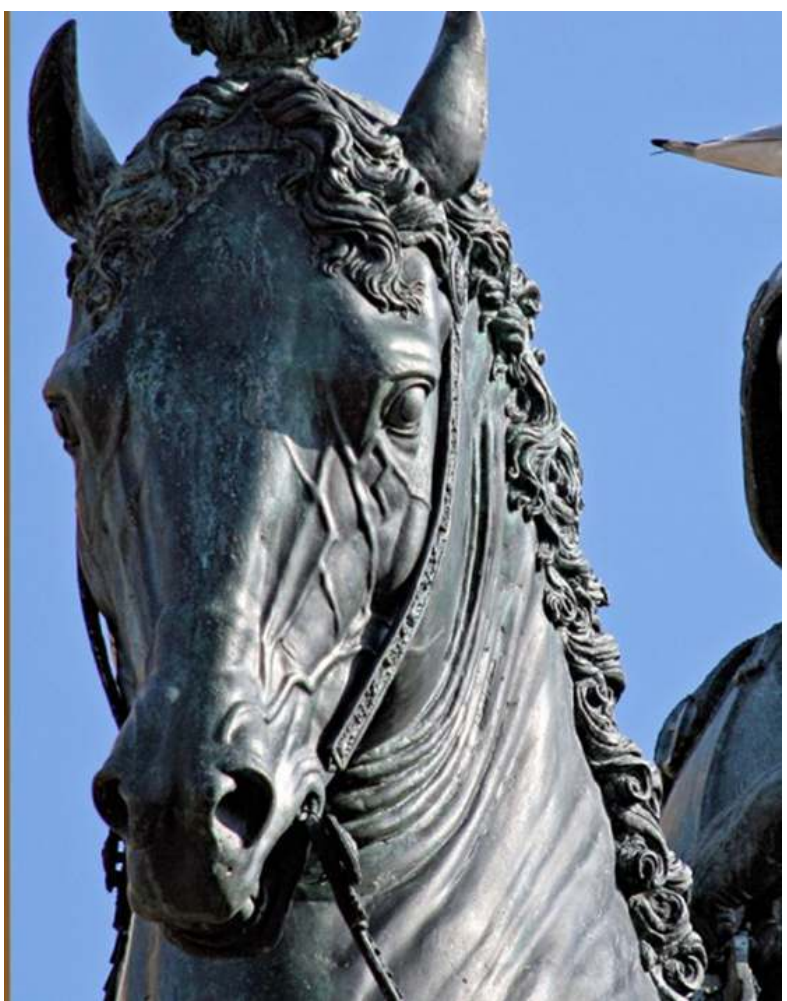

Andrea del Verrocchio puis Alessandro Leopardi (signé), détail de la Statue équestre de Bartolomeo Colleoni, ronde-bosse, bronze. Venise, Campo San Giovanni e Paolo, in situ, 1479-1488 puis 1496 (documenté).

Phot. Fémelat, Armelle. ㄷ Armelle Fémelat, 2005.

Derniers détails naturalistes de la morphologie et de la physiologie chevalines remarquables des effigies équestres italiens des Trecento et Quattrocento, les veines affleurantes et les plis de la peau.

\section{Veines affleurantes et plis de peau}

Comme l'illustrent les deux célèbres statues équestres du Gattamelata ${ }^{42}$ et du Colleoni ${ }^{43}$ (fig. 10), nombre de montures d'effigies équestres montrent des plis de peau, des jeux de veines et de muscles. Invariablement situés au niveau de la tête, de l'encolure et de la jonction entre le corps et les membres, de tels détails semblent avant tout destinés à figurer les mouvements de l'animal et donc à transmettre sa pulsion de vie. Ils participent de son incarnation sans la moindre dimension symbolique. 
Figure 10

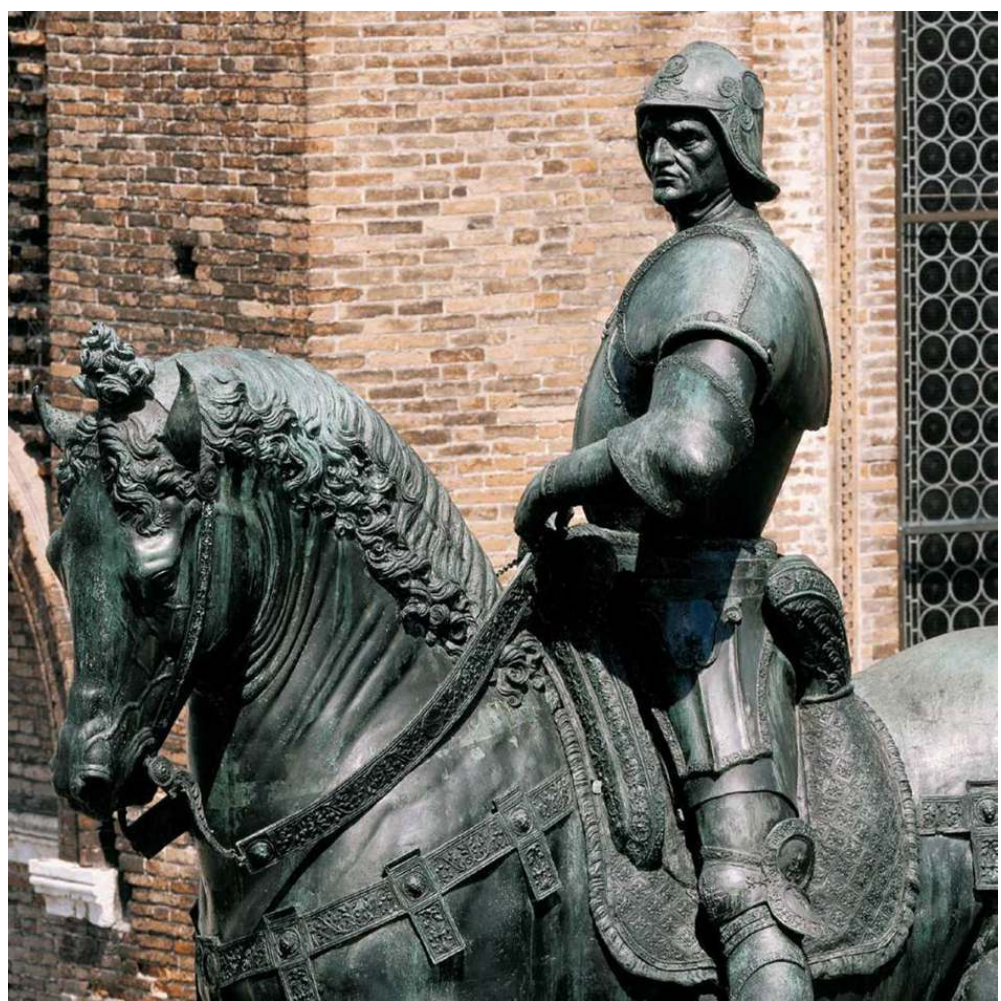

Andrea del Verrocchio puis Alessandro Leopardi (signé), tête et encolure de la Statue équestre de Bartolomeo Colleoni, ronde-bosse, bronze. Venise, Campo San Giovanni e Paolo, in situ, 1479-1488 puis 1496 (documenté)

Phot. Fémelat, Armelle. ㄷ Armelle Fémelat, 2005

D'autres détails encore sont à mettre en rapport avec des pratiques hippologiques et hippiatriques des XIV et $\mathrm{XV}^{\mathrm{e}}$ siècles.

\section{Des pratiques hippologiques et hippiatriques}

L'observation des chevaux des portraits équestres et de leurs études préparatoires permet parfois en outre de constater la mise en valeur de certains détails qui sont autant de traces de pratiques hippologiques et hippiatriques contemporaines, en l'espèce les naseaux fendus et les queues mutilées.

\section{La fente des naseaux}

D'origine orientale, la pratique hippiatrique d'entailler les naseaux des équidés pour faciliter leur respiration et améliorer leur performance remonterait à l'Égypte ancienne ${ }^{44}$. Attestée dans la péninsule italienne à la fin $\mathrm{du}_{\mathrm{XIV}}^{\mathrm{e}}$ et $\mathrm{au} \mathrm{XV}{ }^{\mathrm{e}}$ siècle, son usage semble avoir été courant en Europe centrale et orientale des siècles durant. Si aucun portrait équestre n'illustre directement cette pratique, plusieurs dessins de Pisanello en font état ${ }^{45}$ (fig. 11). L'étude préparatoire à la médaille de Jean VIII Paléologue conservée au Louvre ${ }^{46}$ montre ainsi une tête de cheval harnachée aux naseaux très distinctement fendus. Ce même détail se retrouve sur le cheval d'un des écuyers, à l'arrière-plan de la fresque pisanellienne illustrant l'histoire de saint Georges peinte à Sant'Anastasia de Vérone entre 1433 et $1438^{47}$. Ce détail distinctif s'impose donc comme un des moyens picturaux 
choisis par l'artiste pour spécifier les chevaux de type oriental, et plus largement pour évoquer l'Orient - légendaire ou réel selon les représentations - tout en s'inscrivant dans une tradition picturale préétablie puisque des chevaux aux naseaux fendus furent peints dans le nord de la péninsule dès les dernières années du Trecento ${ }^{48}$.

Figure 11

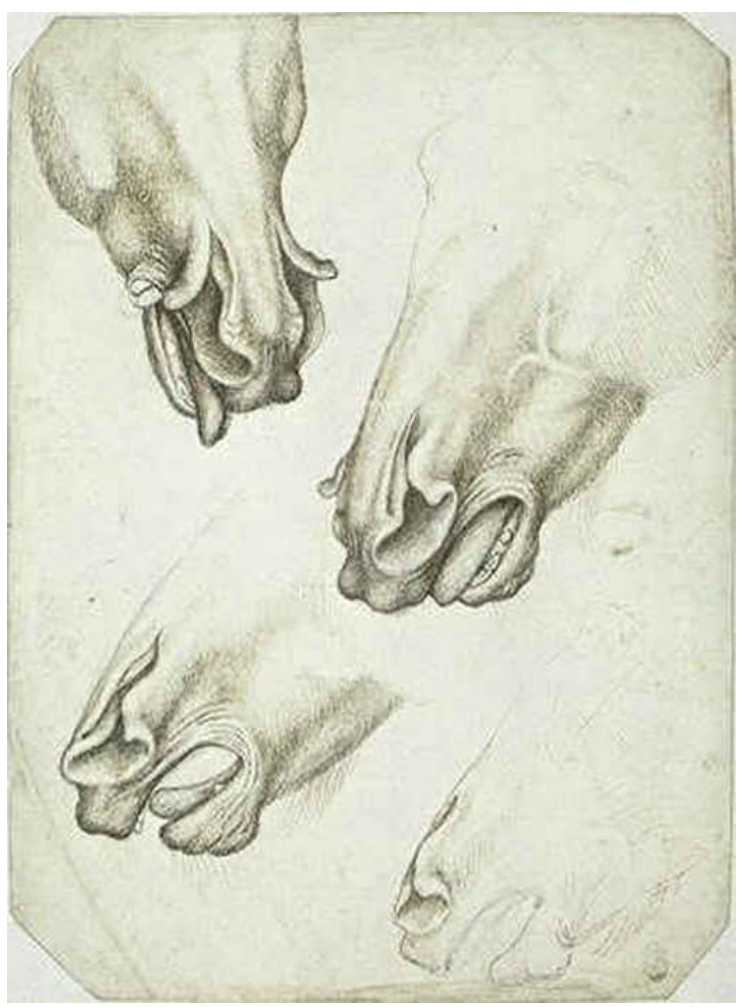

Attribuée à Pisanello, Étude de quatre museaux et naseaux fendus, plume et encre brune sur papier filigrané. Paris, musée du Louvre, département des Arts graphiques, Inventaire 2353, ca 1435-1445 Phot. Fémelat, Armelle. (c) Armelle Fémelat,

Pour sa part, Léonard dénonça cette pratique. Dans une note du Codex Atlanticus, il affirme sévèrement que «l'usage de trancher les naseaux des chevaux mérite d'être tourné en dérision. Les imbéciles observent la coutume quasiment comme s'ils croyaient que la nature manquât du nécessaire et eût besoin d'être corrigée par les hommes ${ }^{49}$. Probablement apparues dans la péninsule Ibérique au XIV siècle, les pratiques de mutilation des oreilles et de la queue des équidés firent également l'objet de critiques sévères dans l'Italie de la Renaissance ${ }^{50}$.

\section{La caudectomie}

Aux $\mathrm{XIV}^{\mathrm{e}}$ et $\mathrm{XV}^{\mathrm{e}}$ siècles, les chevaux de combat et les montures de chasse eurent fréquemment la queue attachée court, voire sectionnée. Cela pour des raisons de commodité évidentes : éviter que les hampes des lances ou les lames des épées ne se prennent dedans. Les montures des combattants de la porte de bronze du Castelnuovo de Naples - sculptée sous la direction de Guglielmo Lo Monaco entre 1474 et 1477, et qui illustre la guerre ayant opposé Ferrante d'Aragon à Jean d'Anjou entre 1458 et $1462^{51}$ affichent ainsi presque toutes une queue coupée ou attachée très court. De même, la queue des trois chevaux qui figurent au centre et dans la partie droite du panneau 
florentin des Batailles de San Romano de Paolo Uccello ${ }^{52}$ a été coupée très courte. La finalité de la caudectomie équine fut parfois aussi motivée par des critères esthétiques, permettant ainsi d'«éviter que le cheval ne fouaille de façon inopportune et disgracieuse ». Ce faisant, comme le précise Elisabetta Deriu, «contrairement à la mutilation des oreilles, elle marque plutôt l'adhésion à un idéal très répandu en milieu curial, et selon lequel le cheval doit faire preuve de grâce et de discipline, tout comme son cavalier $»^{53}$.

Différentes composantes morphologiques, physiologiques et traces de pratiques hippologiques et hippiatriques mutilantes comptent donc parmi les détails significatifs des effigies équestres des Trecento et Quattrocento. Figurés avec un soin particulier, ils sont destinés à attirer l'attention du spectateur, tant pour ce qu'ils représentent que pour la manière dont ils sont représentés. De tels détails évoquent en effet autant la réalité physique et physiologique de l'animal que sa valeur symbolique dans la culture et l'imaginaire collectif de l'époque, et que le talent de l'artiste, l'enjeu de ce type de figuration étant notamment de nature artistique. Ainsi, le portrait équestre participa pleinement de la «révolution naturaliste » qui engendra, à la Renaissance, un nouveau regard sur le monde et un renouvellement des pratiques artistiques. Certains artistes manifestèrent en particulier une curiosité quasi "scientifique » à l'égard des animaux, qui les amena à les observer et à les dessiner d'après nature, de manière inédite. De fait, nombre d'études naturalistes de chevaux, souvent réalisées d'après nature - parfois même d'après modèle vivant - sont contemporaines des effigies équestres des $\mathrm{XIV}^{\mathrm{e}} \mathrm{et} \mathrm{XV}^{\mathrm{e}}$ siècles, qu'elles en aient été des études préparatoires ou non.

\section{Les études d'après nature : ad vivum}

31 "Dessine les animaux d'après nature aussi souvent que tu le peux ", conseillait Cennino Cennini vers 1390 dans son Libro dell'arte ${ }^{54}$. De fait, dès la fin du XIve siècle, des artistes s'adonnèrent à la pratique du dessin d'après nature, dite ad vivum ou ad vivo. Étape essentielle de la tendance au naturalisme, une telle pratique - qui s'insérait dans une tendance plus générale d'observation et d'investigation de la nature - apparut dans le nord de l'Italie à la fin du Trecento et ne cessa de se développer au fil des décennies et des siècles ${ }^{55}$. Giovannino de'Grassi, Jacopo Bellini, Pisanello et Léonard de Vinci comptent parmi les principaux artistes du Quattrocento qui se passionnèrent pour la représentation équine. Multiples, leurs études font preuve d'un incontestable souci de réalisme, ce qui laisse supposer qu'elles furent réalisées ad vivo ${ }^{56}$ même si la prudence reste de mise. En effet, s'agissant des études comme des effigies équestres, il s'avère souvent impossible de savoir si les artistes ont réellement travaillé d'après modèle vivant faute de source. Dévolues à l'ensemble du corps de l'animal - du point de vue du modèle, des proportions ou du mouvement - ou à certains détails anatomiques précis, de telles études corroborent l'avis des manuels d'hippologie ${ }^{57}$ selon lequel l'examen de l'aspect extérieur d'un cheval doit procéder du général au particulier.

Selon Daniel Arasse, la place croissante accordée aux études de détails constitue une des particularités remarquables de l'évolution de la pratique artistique entre le $\mathrm{XIV}^{\mathrm{e}}$ et le $\mathrm{Xv}^{\mathrm{e}}$ siècle en Italie ${ }^{58}$. La spécificité des études chevalines tient en outre à leur diversité, avérée tant dans la forme que dans la finalité, les unes constituant de véritables études préparatoires à une représentation équestre précise, les autres étant faites pour ellesmêmes, éventuellement insérées dans un cahier de motifs. Et Arasse de remarquer qu' 
outre leur minutie et leur caractère parfois acharné, les études de détails révèlent que c'est souvent, en définitive, moins la vérité de la représentation en elle-même qui retient alors l'artiste que sa vérité en peinture, en fonction de son intégration dans l'ensemble où ce détail s'insère ${ }^{59}$. zoologiques, sans prise en compte ni du caractère ni du comportement de l'animal ${ }^{66}-$, les animaux sont décrits par types, dans le respect de normes conventionnelles prédéterminées. Ils sont toujours figurés isolés, de profil, dotés de tous leurs détails caractéristiques et assujettis à des schèmes de stylisation précis. Leurs auteurs, des artistes lombards du Trecento finissant, travaillèrent fréquemment à partir d'animaux morts, qui leur facilitaient une observation rapprochée et le choix d'une posture ${ }^{67}$. Puis, dans la première moitié $\mathrm{du} \mathrm{XV}^{\mathrm{e}}$ siècle, Pisanello franchit une étape décisive avec ses nombreuses études ad vivo, consacrant ainsi la primauté du disegno sur la destination iconographique de l'image. Par ailleurs, il innova également en proposant de restituer la morphologie de l'animal et sa façon de se mouvoir par le prisme de techniques graphiques adaptées ${ }^{68}$. Dans la seconde moitié du siècle, Léonard de Vinci poussa encore plus loin ces investigations. N'ayant de cesse d'interroger les mécanismes inhérents à la réalité physionomique chevaline, incluant l'anatomie et l'étude de la structure musculaire, il tira lui aussi parti des ressources propres de médiums graphiques diversifiés - et inédits pour certains d'entre eux comme la sanguine.

\section{Les études morphologiques de Pisanello}

36 La comparaison des chevaux dessinés par Pisanello avec ceux antérieurs de quelques décennies du Taccuino dei disegni donne à voir l'évolution des conceptions et des pratiques artistiques en matière de représentation équine. En effet, le Taccuino bergamasque ne montre que des chevaux entiers et exclusivement de profil, à la différences des équidés pisanelliens figurés selon des points de vue variés, en totalité ou seulement en partie, en bonne santé ou malade, harnachés ou non, selon une grande variété de types et de modèles. Si ces deux séries d'études équines se rejoignent dans leur souci d'exactitude et dans le rendu des détails et des textures, celles de Pisanello surpassent celles du Taccuino par la finesse de l'observation morphologique retransmise et par leur subjectivité. 

par Pisanello ${ }^{69}$ (fig. 12), seul l'âne pisanellien rend perceptible le sens de l'esquisse de son auteur, ainsi que sa manière de procéder - par décomposition analytique et par réitération. Le motif principal situé au centre de la feuille est en effet complété, dans le coin inférieur gauche, par le détail de la croupe et de la queue ainsi que par un croquis sommaire du baudet. De plus, au-delà du type morphologique, Pisanello a su rendre le caractère de l'animal par le biais de sa silhouette, «de l'attitude servile de son port de tête, de l'expression de l'œil, au mouvement soumis des oreilles " pour reprendre les termes de Dominique Cordellier ${ }^{70}$. Un tel procédé était totalement étranger à ses prédécesseurs lombards et pavesans, encore largement tributaires des conventions figuratives gothiques, et qui, s'ils exprimèrent un souci d'imitation de la nature évident, ne firent pas montre de la moindre subjectivité.

Figure 12

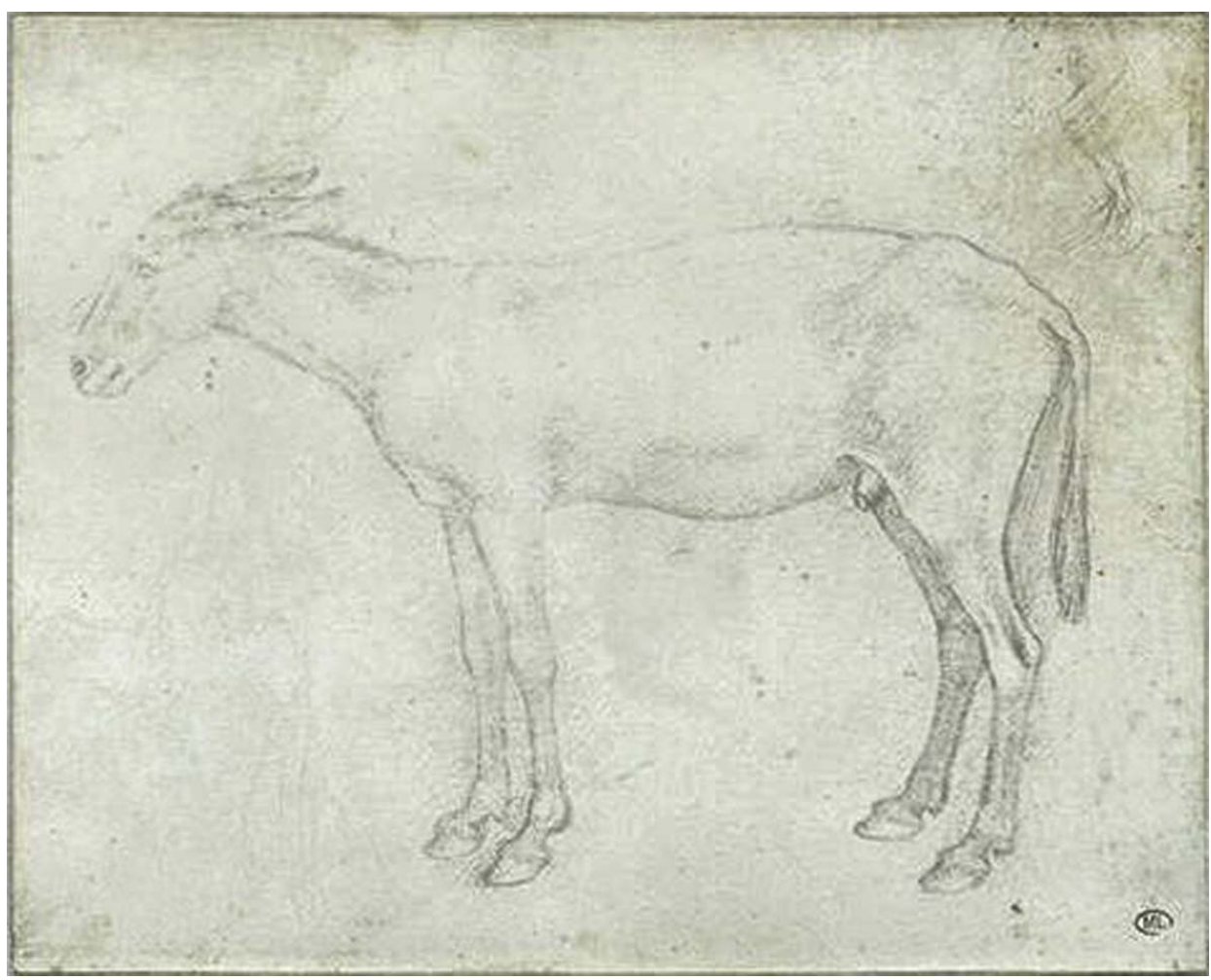

Attribuée à Pisanello, Étude d'un âne de profil gauche, avec l'esquisse d'un âne et d'un ânier, la reprise de sa croupe et de sa queue. Paris, musée du Louvre, département des Arts graphiques, Inventaire 2458, années 1430.

Phot. Fémelat, Armelle. (c) Armelle Fémelat, 2005

Comme l'illustrent également plusieurs dessins de têtes de chevaux harnachés réalisés dans l'atelier du maître, la méthode pisanellienne consistait en une retranscription analytique graphique à partir d'une observation sur le modèle vivant. Ainsi l'œuvre graphique de Pisanello matérialise-t-elle une étape importante dans l'évolution des représentations équines, et plus largement dans l'évolution artistique décisive qui se joua en Italie dans la première moitié $\mathrm{du} \mathrm{xv}^{\mathrm{e}}$ siècle. Dans le domaine spécifique de la représentation des chevaux d'après nature, cette œuvre combine des données complémentaires de morphologie et de mouvement transmises grâce aux techniques 
graphiques les mieux adaptées - qui furent autant de points de départ aux investigations, plus tardives et plus décisives encore, de Léonard.

\section{Les études anatomiques et les recherches dans la représentation du mouvement de Léonard de Vinci}

L'idée persistante selon laquelle Léonard aurait composé un traité sur l'anatomie du cheval, dont il ne reste rien aujourd'hui, remonte à la seconde moitié $d u \mathrm{XVI}^{\mathrm{e}}$ siècle, via Giorgio Vasari et Giampaolo Lomazzo ${ }^{71}$. Et le "Libro di cauallj sc(h)iziati pel cartone ", livre constitué de dessins de chevaux et d'études pour la Bataille d'Anghiari, mentionné dans une note de 1503-1504 du manuscrit Madrid II ${ }^{72}$ semble avoir connu le même sort funeste.

L'essentiel des dessins d'anatomie équine de Léonard daterait de la fin des années 1480 et de la décennie $1490^{73}$, époque à laquelle l'artiste se trouvait à Milan et œuvrait au projet de Statue équestre de Francesco Sforza. Toutefois, dans les années 1507-1510, alors qu'il travaillait au projet de Tombeau équestre de Gian Giacomo Trivulzio, Léonard réinvestit le champ de l'anatomie chevaline, notamment par le biais de l'anatomie comparative: il confronta l'homme au cheval ainsi qu'à d'autres animaux, du point de vue osseux et musculaire ${ }^{74}$. De plus, l'artiste accordait une importance fondamentale à la technique graphique en matière d'investigation du réel ${ }^{75}$, ayant reconnu aux dessins anatomiques une efficacité cognitive supérieure à la pratique même de l'anatomie. Selon lui, de tels dessins auraient permis d'y voir « tous les détails en une seule figure ${ }^{76}$ ». Réalisés d'après nature ou non, figurant l'animal dans son ensemble ou seulement certains détails, les dessins équins léonardiens frappèrent ses contemporains, et ses élèves en particulier, comme l'attestent les nombreuses copies qu'ils en firent ${ }^{77}$.

41 Les sujets et les méthodes de Léonard marquèrent une nouvelle étape dans la représentation du cheval. Ses nombreuses études d'après nature témoignent en effet d'un regard inédit et d'une approche "pseudo scientifique ", incluant des angles de vue variés et complémentaires dans l'étude de l'anatomie, de la musculature et de la décomposition des mouvements. En l'espèce, l'artiste semble avoir hérité de la curiosité et de l'esprit universel de Verrocchio, dans l'atelier duquel il travailla à Florence entre 1469 et 1480 environ. Comme le prouvent ses études de chevaux d'après nature et d'après les modèles antiques, Verrocchio s'intéressa également à la représentation de l'équidé ${ }^{78}$, notamment lorsqu'il travailla à la Statue équestre de Bartolomeo Colleoni entre 1479 et $1488^{79}$. Néanmoins, ses études d'après des animaux réels ne semblent pas avoir eu pour finalité la recherche d'une vérité en matière de représentation, au contraire de Léonard dont les dessins ad vivum lui fournirent les moyens artistiques de rendre convaincante leur idéalisation formelle.

Les commentaires manuscrits qui accompagnent certains dessins léonardiens prouvent qu'il travailla en observant les équidés sur le vif, notamment dans les écuries de Milan ${ }^{80}$. En témoignent ses études du Grand Genêt ${ }^{81}$ et du Sicilien ${ }^{82}$, deux chevaux de l'écurie de Galeazzo Sanseverino, gendre et capitaine général de Ludovic le More, nommé grand écuyer à la Cour de France en 1505. Qui plus est, comme l'indique une note du Codex Forster III, le 16 juillet 1493, Léonard établit une liste des chevaux milanais qu'il avait vus ou dont il avait entendus parler ${ }^{83}$. Au vu des notes et des dessins conservés, ses deux grands axes d'investigation et de représentation du cheval furent les proportions et le mouvement - les deux mêmes grands axes en fonction desquels il aborda le corps humain. La question des proportions participait de sa conception d'un cheval idéal, 
abordée ultérieurement. Quant au mouvement, son expression passait non seulement par le choix des postures et des attitudes, mais également par les techniques graphiques mises en œuvre.

Les chevaux des études préparatoires pour les statues équestres, jamais réalisées, de Francesco Sforza (ca 1484-1490) ${ }^{84}$ et de Gian Giacomo Trivulzio (ca 1508-1511) ${ }^{85}$ trottent ou se cabrent. Cette série de dessins montre bien la manière dont l'artiste focalisa son attention sur la représentation du mouvement, en particulier celui de l'animal. Visiblement, son attention se concentra avant tout sur la posture de l'animal, dont a ensuite découlé la position du cavalier. Le dynamisme qui s'en dégage provient en grande partie de la tension créée par la collision des mouvements des deux protagonistes. Que le cavalier se tienne droit sur un cheval au trot ou penché en avant sur un cheval cabré, le plus souvent, chacun des deux est animé d'un mouvement latéral: l'animal incurve l'encolure d'un côté tandis que l'homme se tourne de l'autre, dans un jeu de double contrapposto.

Les qualités de dessinateur de Léonard et son choix avisé des médiums et des techniques sont également fondateurs de la vitalité de ses chevaux et cavaliers dessinés. De fait, dans les deux études préparatoires pour le projet $S f o r z a^{86}$, la véhémence du ductus linéaire du tracé à la pointe d'argent concourt à exprimer l'impulsion vigoureuse du cavalier, dans une très grande netteté. Même dans certaines études pourtant rapidement esquissées pour le monument Trivulzio, l'accent est toujours mis sur l'insufflation du mouvement, qui apparaît parfois comme le véritable sujet du dessin. Pour ce faire, l'artiste n'hésita d'ailleurs pas à superposer les traits, avec pour conséquence l'accolement de petites variations dans les postures, à l'origine d'un effet dynamique ${ }^{87}$.

Si la généralisation des études naturalistes ad vivum est significative des pratiques artistiques de l'époque, elle ne doit toutefois pas pour autant s'interpréter en termes de progrès. En effet, comme le rappelle Michel Pastoureau

le réalisme animalier de Pisanello [...] n'est qu'une manière parmi d'autres de représenter la nature. Une manière qui a le souci de l'exactitude et du détail descriptif, mais une manière qui n'est pas plus "vraie » que la stylisation expressionniste du bestiaire héraldique ou de la faune allégorique de la poésie animale ${ }^{88}$.

Et il existe un abîme entre la pratique des études anatomiques et le rendu artistique attendu d'un portrait équestre comme le fait valoir la critique émise par Pomponio Gaurico dans son Traité de sculpture paru en 1504, au sujet de la monture de Bartolomeo Colleoni - conçue par Verrocchio entre 1479 et $1488^{89}$ - qu'il estime être une étude anatomique par trop tatillonne, assimilable à un écorchép ${ }^{90}$. Si la question des études naturalistes de chevaux ne se pose pas en termes de vérité, elles n'en donnent pas moins la mesure de l'évolution contemporaine du rapport des artistes à la nature, de leur conception et de leur pratique artistiques. De fait, la finalité d'un tel naturalisme n'était alors pas la vérité équestre mais bien un enjeu artistique, le portrait équestre ou toute autre forme artistique aboutie. Au reste, le naturalisme n'est pas la seule tendance qui caractérise alors l'effigie équestre.

47 Une des spécificités des portraits équestres italiens des Trecento et Quattrocento tient en effet dans leur double tendance au naturalisme et à l'idéalisation. D'une manière générale, cette tension entre individualisation et idéalisation se trouve au cœur de la pensée artistique de la Renaissance, qui fit du portrait son terrain d'élection. De plus, la conception duelle du portrait, qui le place entre réalité et idéalité, entre ressemblance et 
Beauté idéale, est une constante de la culture occidentale. Dans le cas spécifique des portraits officiels, l'enjeu est de trouver un juste équilibre entre la représentation fidèle du modèle et le respect de son statut et de sa dignité - la virtù italienne. Par ailleurs, ni le degré de précision et de rendu des détails, ni l'individualisation physionomique ne gagent de la fidélité du portrait, ils ne constituent pas des critères d'évaluation de sa "véracité ». Car son essence est ailleurs : la vérité du portrait étant de nature artistique, elle ne se confond pas avec celle du personnage représenté - ni de sa monture dans le cas particulier de l'effigie équestre. Ce qui explique aussi sa part d'idéalisation.

\section{Un cheval idéal - une tendance à l'idéalisation}

Faut-il parler de cheval idéal, de chevaux idéaux, ou d'idéal de cheval? Autant de subtilités liées à l'idéalisation qui marquent visiblement le portrait équestre et plus généralement la représentation équestre à la Renaissance. Cette tendance à l'idéalisation du cheval renvoie à l'idée d'un idéal équin, en particulier à l'idéal théorique de Leon Battista Alberti (Traité sur le cheval vivant, 1453) et à l'idéal artistique de Léonard de Vinci (dessins et notes des années 1480-1510) qui entrent en résonance avec un idéal équin littéraire véhiculé depuis la littérature hippiatrique médiévale jusqu'aux traités d'art équestre de la Renaissance en passant par le Sonetto del Cavallo perfetto ${ }^{91}$.

Les littératures agronomiques et hippiatriques du Moyen Âge et de la Renaissance traitèrent immanquablement de l'aspect général du cheval et de ses proportions. Des canons, c'est-à-dire des règles fixant des rapports de proportions idéalement harmonieux, furent élaborés sans jamais cesser d'évoluer. Relayés au Quattrocento par les traités de mascalcia (maréchalerie) - abordant conjointement l'hippiatrie et l'hippologie -, les textes hippiatriques médiévaux instaurèrent une véritable tradition de la description du modèle et des canons d'un cheval idéal. Davantage inspirés des auteurs antiques et de l'observation des corps réels, les canons équins de la Renaissance se sont distingués de leurs précédents médiévaux, encore amplement moralisés par une pensée religieuse.

La question générique des proportions, qui participe de la problématique de la représentation du corps - humain et animal -, a constitué un axe de recherche récurrent chez les penseurs et les artistes de la Renaissance, de même que la question plus spécifique des proportions équines - sur la base d'un cheval idéal. Le graveur allemand Hans Sebald Beham alla jusqu'à dédier un traité spécialement aux proportions équines, publié à Nüremberg en $1528^{92}$, et à illustrer l'édition française du célèbre traité d'hippiatrie de Lorenzo Rusio (1250), parue en $1531^{93}$. Ce faisant, il s'inspira d'Albrecht Dürer, également passionné par la problématique de la représentation et des proportions équines, à l'instar de Leon Battista Alberti et de Léonard, autres grands génies universels de la Renaissance, à la fois penseurs, théoriciens et artistes.

\section{L'idéal théorique de Leon Battista Alberti à travers son Traité sur le cheval vivant}

51 Le Traité sur le cheval vivant (De equo animante) que Leon Battista écrivit en latin vers $1445^{94}$ et qu'il dédia à Lionel d'Este, marquis de Ferrare, est un unicum. Un ovni littéraire et scientifique de la Renaissance. Classique dans la forme, inscrit dans les traditions scientifiques antique et médiévale ${ }^{95}$, ce traité est en revanche unique dans le traitement $\mathrm{du}$ sujet. L'humaniste y a élaboré une synthèse des savoirs de l'époque sur le cheval vivant, abordant des questions aussi vastes que la reproduction, les géniteurs, le poulain, l'éducation, le dressage du cheval de guerre, et moult considérations hippiatriques ${ }^{96}$. 
Resté sans descendance, cet ouvrage n'en constitue pas moins le signe précurseur d'une nouvelle manière de penser et d'écrire le cheval. L'humaniste y propose un idéal esthétique et moral $d u$ "cheval du prince» fondé sur une synthèse du savoir hippologique et hippiatrique antique et médiéval.

Dans ce traité, Alberti évoque le cheval idéal dans ses trois déclinaisons : étalon, c'est-àdire reproducteur, poulinière et poulain, avec un intérêt spécifique pour le premier. Choisi entre mille et objet de tous les soins, le cheval d'élite est selon lui avant tout un cheval de guerre, qui de surcroît ne peut être qu'un mâle entier. Ce "cheval du Prince ", c'est l'hippos polemistès des Grecs et le bellator equus des Latins tel que Pline l'Ancien le décrit dans son Histoire naturelle ${ }^{97}$. Alberti en énumère les qualités, qui se situent tant sur le plan physique (morphologie, capacité physique ou "condition») qu'au niveau esthétique (éloge de sa «beauté ») et du caractère (courage et intelligence, incluant une dimension morale), laissant de côté l'opposition des Anciens entre cheval de guerre et cheval de parade. De fait, pour l'humaniste, le cheval idéal doit, à l'image de son prince de maitre, cumuler les qualités du cheval de guerre contemporain et du cheval de guerre des Anciens, de manière à briller en toutes circonstances, à vertus égales sur le champ de bataille et dans l'apparat de la paix. Dans ce traité, Alberti part de l'esthétique et de la morale du cheval idéal pour arriver à l'évocation des conditions techniques de production, de conservation et de dressage de l'animal, adoptant en cela une méthodologie similaire à celle mise en œuvre dans ses traités dédiés à l'architecture et à la sculpture ${ }^{98}$.

La description albertienne - la plus longue du traité - du géniteur idéal débute par la morphologie d'ensemble et les proportions avant de s'arrêter sur différentes parties de son corps. Sur la base de cette description, des correspondances peuvent s'établir avec certains portraits. Ainsi, selon Elisabeth Oy-Marra ${ }^{99}$, la monture de Niccolò da Tolentino dans la cathédrale de Florence ${ }^{100}$ (fig. 13) partage hauteur et robustesse avec le cheval idéal d'Alberti ainsi qu'une tête moyenne avec de fines oreilles mobiles, des naseaux dilatés, une attache de la tête délicate, une encolure bien implantée sur les épaules, un dos régulier, un poitrail généreux, des membres légers et droits. Et Mary Bergstein ${ }^{101}$ de proposer un paragone poussé entre l'idéal albertien et la monture donatellienne du Gattamelata ${ }^{102}$, tout en évoquant les contacts qui ont pu lier les deux artistes. En réalité, il est impossible de savoir si ces artistes de l'avant-garde florentine eurent accès au traité albertien sur le cheval vivant, ou au moins à ses idées; néanmoins, l'équidé est indéniablement au cœur de leurs recherches, a fortiori pour les artistes chargés de concevoir un portait équestre, à l'instar de Léonard ${ }^{103}$. 
Figure 13

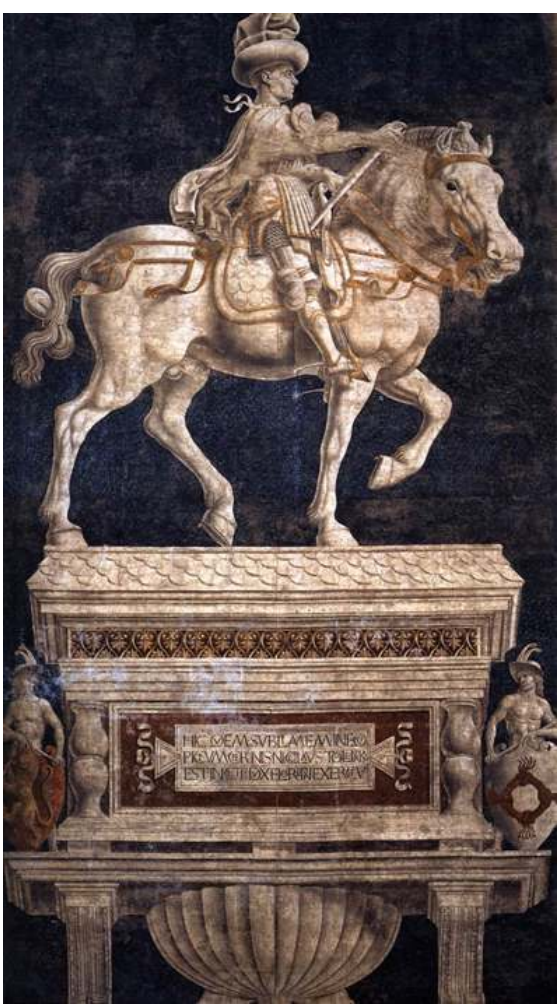

Andrea del Castagno (documenté), Monument funéraire en trompe-l'œil de Niccolò da Tolentino, fresque. Florence, Santa Maria del Fiore, 1456 (documenté).

Phot. Fémelat, Armelle. (C) Armelle Fémelat, 2003.

\section{L'idéal artistique de Léonard de Vinci à travers ses dessins et ses notes} sphère artistique, à la différence d'Alberti qui œuvra dans le champ théorique. Dès ses premières études préparatoires pour l'Adoration des Mages ${ }^{104}$ dans les années $1478-1480^{105}$, Léonard investit la problématique des proportions équines, auxquelles il consacra quantité de notes et de dessins, en parallèle à des recherches dédiées aux proportions du corps humain ${ }^{106}$.

Ses croquis annotés des proportions de tout ou partie du corps de l'équidé (de la tête ${ }^{107}$ et des membres ${ }^{108}$ notamment) rendent compte de la manière dont il s'employa à définir le type morphologique du cheval idéal, en digne héritier de Verrocchio. Le concepteur de la Statue équestre de Bartolomeo Colleoni ${ }^{109}$ a en effet lui aussi laissé deux études des proportions du corps du cheval incluant commentaires et mesures ${ }^{110}$. Une telle méthode figurative - avec inscription d'indications et de calculs à l'intérieur ou autour de la silhouette de l'animal - reprise par Léonard et qu'illustrent plusieurs dessins ${ }^{111}$, était vouée à l'établissement d'un système cohérent de proportions issu du système vitruvien ${ }^{112}$. Établi sur l'unité de mesure de la tête de cheval - testa -, subdivisée en grado - un sixième de tête - et en minuto - un sixième de grado -, eux-mêmes subdivisés en minimo ou en seminuto, etc. ${ }^{113}$, le cheval artistique idéal de Léonard apparaît comme la manifestation et l'aboutissement d'un système de composition et de calcul rationnalisés. L'artiste a ainsi reproduit différents types de chevaux dans le but d'établir un paradigme complet du 
modèle équin, l'observation d'individus réels - dans leur diversité - ayant servi de base à ses calculs savants et à l'élaboration d'un cheval artistique idéal.

Bien que réalisées d'après des modèles vivants - au regard des proportions naturelles, de l'anatomie, de la morphologie, de la physiologie et du mouvement -, les recherches léonardiennes en matière équine eurent bien pour finalité la conception et la représentation d'un cheval idéal, et non celle de la reproduction fidèle de la réalité pour elle-même. Les croquis, dessins et annotations qui nourrirent et construisirent ces recherches illustrent bien la manière dont l'artiste fit son miel de l'observation de la nature. Pour lui, observer et comprendre les chevaux furent des étapes aussi cruciales que fondatrices, mais jamais une finalité : elles lui servirent à concevoir ses propres chevaux et un cheval idéal. Ses chevaux dessinés sont ainsi « représentés avec objectivité, une image fidèle d'après nature, mais toutefois idéalisée selon un concept classique de la beauté » comme le résume Carlo Pedretti ${ }^{114}$. Fondant la beauté artistique sur la science de la nature, le créateur se mit en quête de réunir les beautés naturelles éparses pour atteindre un modèle plus parfait, rejoignant en cela les préceptes développés par Alberti dans ses traités sur la peinture et la sculpture ${ }^{115}$, et entérinant le constat de Guido Perocco selon lequel

le cheval apparaît comme une forme idéale, symbole de force, d'énergie contenue, de puissance guidée par la raison et par la souveraine vigueur de l'esprit; l'incarnation en un mot d'un idéal esthétique dont l'esprit humain dote la beauté et la noblesse d'un destrier, qui devient partie intégrante de la vie de l'homme dans une rencontre entre la souveraineté et l'idée et la réalité contingente ${ }^{116}$.

57 Cette analyse des chevaux des portraits équestres italiens des Trecento et Quattrocento, incluant une confrontation avec des études d'après nature et toute une production théorique, met en exergue la double distance exprimée par de telles représentations : en plus d'être - plus ou moins - historiques et naturalistes, elles sont une expression artistique. L'expression artistique de la vision combinée d'un créateur et d'un commanditaire, conçue en fonction d'un public prédéterminé. À ce titre, elles s'intègrent à la problématique plus vaste de la culture équestre de l'époque et du milieu qui les a produites. La réalité équestre a nourri et inspiré les protagonistes de tels portraits, en même temps que ces images ont permis aux acteurs de la société cavalière de la Renaissance de s'interroger et le cas échéant d'évoluer. De fait, dans l'Italie de la Renaissance qui voit l'avènement de l'art équestre, le cheval est à la fois acteur, moyen et symbole.

«Marqueur social », symbole du pouvoir et de la puissance militaire, l'équidé constitue un indicateur des choix opérés à l'intérieur du système figuratif de la représentation du pouvoir : il est une forme "signifiante " utilisée non seulement comme qualificatif et comme élément de faire-valoir, mais encore comme outil épidéictique au service de la célébration de son cavalier. Et dans l'effigie équestre, qui est un instrument de communication politique, l'équidé est résolument au service de l'homme, au sens propre comme au sens figuré, à la fois piédestal et faire-valoir. Dans ce contexte, la double tendance - au naturalisme et à l'idéalisation - à l'œuvre dans la représentation du cheval contribue à la représentation globale, et en l'occurrence à la glorification du cavalier.

Le mouvement d'individualisation qui caractérise la Renaissance est à l'œuvre, et il a aussi progressivement concerné certains animaux, à commencer par la plus noble conquête de l'homme. De "véritables portraits de chevaux", affranchis de tout cavalier, apparurent dès le début du Cinquecento, qui fut aussi le siècle de l'ouverture des 
premières académies d'art équestre. En particulier à Mantoue, cité équestre entre toutes, où furent peints les Étalons préférés de Frédéric II de Gonzague par Jules Romain dans le salon des Chevaux du palais du Tè ${ }^{117}$, d'autres étalons du même duc dans des tableaux malheureusement perdus ${ }^{118}$ ainsi que dans les peintures murales disparues de la chambre des Chevaux du palais ducal ${ }^{119}$. Enfin, un manuscrit entier, dédicacé au duc Frédéric II, fut dédié aux vainqueurs Gonzague des courses de palii, et orné de trente-cinq " portraits » de chevaux des écuries ducales réalisés par Silvestro da Lucca dans des paysages mantouans peints par Lauro Padovano ${ }^{120}$. Ou comment l'on passa du portrait équestre au portrait équin. Changement formel notable mais sans implication signifiante: même privé de cavalier, l'animal donné à voir ne l'est qu'en tant qu'émanation du prince, objet indirect de la représentation.

\section{NOTES}

1. - FÉMELAT, Armelle. Le portrait équestre italien de la fin du Moyen Âge au début de la Renaissance. Thèse d'histoire de l'art moderne. Tours : université François Rabelais, 2010.

2. - Anonyme, Statue équestre du monument funéraire de Ladislas d'Anjou-Duras, ronde-bosse, marbre. Naples, San Giovanni a Carbonara, in situ, 1428 (daté)-ca 1432.

3. - Cola Rapicano (documenté), Portrait équestre de Ferrante d'Aragon, enluminure. Paris, Bibliothèque nationale de France, département des Manuscrits occidentaux, Ms Lat. 12947, f. 2, 1471 (daté). Attribué à Cola Rapicano, Portrait équestre de Ferrante d'Aragon, enluminure. Vienne, Österreichische Nationalbibliothek, Ms 4, f. 3 v ${ }^{\circ}$, ca 1484.

4. - Simone Martini (documenté), Guidoriccio da Fogliano, fresque. Sienne, palais public, salle du Conseil, in situ, ca 1330.

5. - Anonyme, Statue équestre de Cangrande Della Scala, ronde-bosse, pierre. Vérone, musée de Castelvecchio, ca 1340-1345: provenant de son monument funéraire au cimetière Scaliger attenant à Santa Maria Antica de Vérone.

6. - Anonyme, Statue équestre de Mastino II Della Scala, ronde-bosse, pierre. Vérone, musée de Castelvecchio, ca 1350 : provenant de son monument funéraire au cimetière Scaliger attenant à Santa Maria Antica de Vérone.

7. - FRANCHINI, Maria. MARESCA, Giuseppe. La fabuleuse histoire du cheval napolitain. Paris : Zulma, 2003.

8. - Pisanello (signé), Médaille à l'effigie de Jean VIII Paléologue, bas-relief, ca 1438-1439.

9. - Unanimement attribuée à Pisanello, Étude avec Jean VIII Paléologue à cheval de profil droit, une encolure de cheval et trois autres figures, plume et encre brune sur papier. Paris, musée du Louvre, département des Arts graphiques, Inventaire MI 1062, ca 1438. Unanimement attribuée à Pisanello, Étude avec un cavalier de profil gauche, trois figures et un cimeterre, plume et encre brune sur papier filigrané. Washington, The Art Institute, Margaret Day Blake Collection, 1961.331, ca 1438.

10. - Attribuée à Pisanello, Étude d'une tête et d'une encolure de cheval oriental aux naseaux fendus vu de trois-quarts, plume et encre brune, tracé préparatoire à la pierre noire sur papier filigrané. Paris, musée du Louvre, département des Arts graphiques, Inventaire 2363, ca 1438. 
11. - Attribuée à Pisanello, Étude d'un cheval oriental aux naseaux fendus vu de trois-quarts face et de trois-quarts dos, plume et encre brune, lavis gris et brun, rehauts de blanc. Paris, musée du Louvre, département des Arts graphiques, Inventaire 2468, ca 1438.

12. - Voir WEIZSÄCKER, Heinrich. "Das Pferd in der Kunst des Quattrocento ». Jahrbuch der Königlischen und Kaiserlichen Kunstsammlungen, 1886, vol. 7, p. 52-61; VICKERS, Michael. "Some preparatory Drawings for Pisanello's Medaillon of John VIII Paleologus ». The Art Bulletin, 1978, vol. LX, n³, p. 417-424; RUGOLO, Ruggero. «Les médailles. Pisanello médailleur : l'humanisme chevaleresque ». Dans PUPPI, Lionello. Pisanello. Paris : Hazan, 1996, p. 145.

13. - JUREN, Vladimír. «À propos de la médaille de Jean VIII Paléologue par Pisanello ». Revue numismatique, 1973, vol. VI, $\mathrm{n}^{\circ} 15$, p. 219-225.

14. - Costanzo da Ferrara (signé), Médaille à l'effigie de Mehmet II, bas-relief, bronze. Washington, National Gallery of Art, Samuel H. Kress Collection, A839-102A, datable de l'hiver 1480-1481.

15. - Voir BLOMAC, Nicole de, BOGROS, Denis. L'Arabe, premier cheval de sang. Paris: CrépinLeblond, 1978.

16. - Donatello (signé), Statue équestre du Gattamelata, ronde-bosse, bronze. Padoue, place du Santo, in situ, 1447-1453 (documenté).

17. - Anonyme, Portrait équestre funéraire d'Antonio Rido, haut-relief, marbre. Rome, Santa Francesca Romana, in situ, ca 1450-1480.

18. - Anonyme, Portrait équestre funéraire de Giordano Orsini, haut-relief, marbre. Monterotondo, Santa Maria delle Grazie, in situ, ca 1484.

19. - Andrea del Verrocchio puis Alessandro Leopardi (signé), Statue équestre de Bartolomeo Colleoni , ronde-bosse, bronze. Venise, Campo San Giovanni e Paolo, in situ, 1479-1488 puis 1496 (documenté).

20. - FÉMELAT, Armelle, op. cit.

21. - Attribuée à Pisanello, Étude d'un cheval couché sur le flanc gauche de profil droit, pointe de métal et rehauts de blanc oxydés sur papier préparé gris bleuté. Paris, musée du Louvre, département des Arts graphiques, Inventaire 2371, ca 1414-1415. Attribuée à Pisanello, Étude d'un cheval harnaché de trois-quarts dos, plume et encre brune, tracé préparatoire à la pierre noire sur papier. Paris, musée du Louvre, département des Arts graphiques, Inventaire 2378, ca 1435. Attribuée à Pisanello, Étude d'un cheval de trois-quarts dos à gauche avec la queue nouée, plume et encre brune, tracé préparatoire à la pointe de plomb sur papier, musée du Louvre, département des Arts graphiques, Inventaire 2444, ca 1435. Attribuée à Pisanello, Étude d'une arrière main de cheval de profil droit, plume et encre brune, lavis beige et aquarelle, tracé préparatoire au stylet sur papier, musée du Louvre, département des Arts graphiques, Inventaire 2366, ca 1435-1445. Attribuée à Pisanello, Étude d'un cheval harnaché de trois-quarts droit, plume et encre brune, tracé préparatoire à la pierre noire sur papier, musée du Louvre, département des Arts graphiques, Inventaire 2391 verso, ca 1435. Unanimement attribuée à Léonard de Vinci, Étude de chevaux maigres vus de dos, pointe d'argent sur papier préparé pourpre. Windsor Castle, Royal Library, RL 12308, ca 1480. Unanimement attribuée à Léonard de Vinci, Étude d'un étalon de profil droit et de croupes de chevaux, plume et encre, plomb sur papier. Windsor Castle, Royal Library, RL 12324, ca 1480.

22. - Attribuée à Pisanello ou à un anonyme lombard, Étude d'un cheval de profil gauche, d'un antérieur, de deux postérieurs et des attributs sexuels mâles de cheval, pointe d'argent sur papier préparé gris. Paris, musée du Louvre, département des Arts graphiques, Inventaire 2367, ca 1415. Unanimement attribuée à Léonard de Vinci, Étude de l'arrière main et des parties génitales mâles d'un cheval, plume et encre, pierre noire sur papier préparé orange. Windsor Castle, Royal Library, RL 12306, ca 1510. Unanimement attribuée à Léonard de Vinci, Étude de l'arrière main de chevaux, plume et encre sombre, pierre noire et bistre sur papier. Windsor Castle, Royal Library, RL 12313, ca 1508-1511. Unanimement attribuées à Léonard de Vinci, Trois études de l'arrière main et de parties génitales mâles de chevaux, pierre noire sur papier blanc. Windsor Castle, Royal Library, RL 12314 , ca 1510. 
23. - Voir PRÉVOST, Brigitte, RIBÉMONT, Bernard. Le cheval en France au Moyen Âge. Orléans : Paradigme, 1994, p. 191.

24. - DERIU, Elisabetta. Le cheval et la cour. Pratiques équestres et milieux curiaux, Italie et France (milieu $d u X^{e} v^{e}$ milieu $d u X_{X I I}{ }^{e}$ siècle). Thèse d'histoire moderne. Paris : Université Paris XII-Paris Est, 2008 , p. 321.

25. - Ibid., p. 322-326.

26. - Gianfrancesco Enzola da Parma (signé), Revers de médaille à l'effigie de Costanzo Sforza, basrelief, 1473 (daté). Gianfrancesco Enzola da Parma (signé), Revers de médaille à l'effigie de Costanzo Sforza, bas-relief, 1475 (daté).

27. - Anonyme, Portrait équestre funéraire d'Annibale Bentivoglio, haut-relief, terre cuite polychrome. Bologne, San Giacomo Maggiore, chapelle Bentivoglio, in situ, 1458.

28. - Commandée à Léonard de Vinci en 1503 pour la nouvelle salle du Grand Conseil de la République du Palazzo Vecchio de Florence, la fresque de la Bataille d'Anghiari n'a jamais été menée à terme.

29. - Unanimement attribuée à Léonard de Vinci, Étude d'un cheval cabré et de têtes, plume et encre sur papier. Windsor Castle, Royal Library, RL 12326, ca 1504-1506.

30. - Anonyme, Cheval de bronze, ronde-bosse, bronze, provenant d'un quadrige, découvert en 1739 dans la zone du forum d'Herculanum. Naples, Museo Nazionale Archeologico, Inventaire 4904, époque claudienne. Anonyme, Tête de cheval, identifiée comme provenant d'un quadrige du théâtre antique d'Herculanum, ronde-bosse, bronze. Naples, Museo Nazionale Archeologico, Inventaire 115391, époque claudienne. Anonyme, Statue équestre de Domiziano Nerva, provenant de Sacellum des Augustales de Misène, ronde-bosse, bronze. Naples, Museo Nazionale Archeologico, Inventaire 155743, dernières années du règne de Domitien.

31. - Art grec, Protomé équin dit Médicis, ronde-bosse, bronze. Florence, Museo Archeologico Nazionale, Inventaire 1639, milieu du IV siècle av. J-C. Voir CIANFERONI, Giuseppina Carlotta. « Notice V.I ». Dans BORMAND, Marc, PAOLOZZI STROZZI, Beatrice. Le Printemps de la Renaissance. La sculpture et les arts à Florence, 1400-1460. Milan/Paris : Officina Libraria/Musée du Louvre, 2013, p. 362-363.

32. - Unanimement attribué à Donatello, Protomé équin dit Carafa, ronde-bosse, bronze. Naples, Museo Archeologico Nazionale, Inventaire 4887, ca 1455. Voir CISERI, Ilaria. « Notice V.II ». Dans BORMAND, Marc, PAOLOZZI STROZZI, Beatrice, op. cit., p. 364-365.

33. - Bonino da Campione (signé), Statue équestre de Bonino da Campione, ronde-bosse, marbre. Milan, Castello Sforzesco, ca 1355-1360.

34. - DE VECCHI, Pier Luigi. «Introduction ». Dans VERGANI, Graziano Alfredo. L'Arca di Bernabo Visconti al Castello Sforzesco di Milano. Milan : Silvana editoriale, Amilcare Pizzi, 2001, p. 15.

35. - Ibid., p. 126.

36. - Notamment : unanimement attribuée à Léonard de Vinci, Tête de cheval tracée au revers du panneau de Sainte Anne, la Vierge et l'Enfant, huile sur bois. Paris, musée du Louvre, département des Peintures, ca 1503-1519.

37. - Études de chevaux conservées à la Royal Library de Windsor Castle : RL 12342, 12343, 12354, $12355,12357,12358,12359,12360$.

38. - Milan, Biblioteca Ambrosiana, Codex Atlanticus, f. 76 recto-a.

39. - ALBERTI, Leon Battista. De equo animante. Éd. et trad. française : BORIAUD, Jean-Yves. Paris : Les Belles Lettres, 1999, p. 18-19.

40. - Ibid., p. 38-39.

41. - Voir n. 19.

42. - Voir n. 16.

43. - Voir n. 19.

44. - Cet usage d'entailler les naseaux des chevaux est précisément décrit par Buffon en 1753, et était encore pratiqué en Islande, dans l'Afrique saharienne et au Turkestan oriental à la fin du XIX 
e siècle : voir HILL, George Francis. Dessins de Pisanello. Paris, Bruxelles : Van Oest, 1929, p. 18, 41 ; CLUTTON-BROCK, Juliet. Horse Power. A History of the Horse and the Donkey in Human Societies. Cambridge : Harvard University Press, 1992, p. 77.

45. - Attribuée à Pisanello, Étude de tête et d'encolure de cheval oriental aux naseaux fendus vu de troisquarts, plume et encre brune, tracé préparatoire à la pierre noire sur papier filigrané. Paris, musée du Louvre, département des Arts graphiques, Inventaire 2363, ca 1438. Attribuée à Pisanello, Étude d'un cheval oriental aux naseaux fendus vu de trois-quarts face et de trois-quarts dos, plume et encre brune, lavis gris et brun, rehauts de blanc. Paris, musée du Louvre, département des Arts graphiques, Inventaire 2468, ca 1438. Attribuée à Pisanello, Tête de cheval oriental aux naseaux fendus vu de face, pointe de métal sur parchemin. Paris, musée du Louvre, département des Arts graphiques, Inventaire 2405, années 1440. Attribuée à Pisanello, Étude de quatre museaux et naseaux fendus, plume et encre brune sur papier filigrané. Paris, musée du Louvre, département des Arts graphiques, Inventaire 2353, ca 1435-1445.

46. - Voir n. 9.

47. - Unanimement attribué à Pisanello, Saint Georges et la princesse, fresque rentoilée. Vérone, Sant'Anastasia, chapelle Giusti, in situ, ca 1433-1438.

48. - Voir notamment une illustration du cinquième chant de la Thébaïde enluminée du Stace (Dublin, Chester Beatty Library, Ms 76, f. 54 vo) attribuée à Jacopo Avanzi ; une fresque attribuée à l'entourage du Maître de l'Adoration des Mages et conservée dans une collection privée de Bergame (citée par CORDELLIER, Dominique. Pisanello, le peintre aux sept vertus. Paris : Réunion des musées nationaux, 1996, p. 216) ; Fieramont à la cour du roi Arthur, enluminure du manuscrit Guiron le Courtois peinte par un Lombard vers 1390 (Bibliothèque nationale de France, Ms Fr. Nouvelle Acquisition 5243, f. $2 \mathrm{v}^{\circ}$ ); des fresques de Giovanni da Modena dans la chapelle Bolognini de San Pietro à Bologne.

49. - Milan, Biblioteca Ambrosiana, Codex Atlanticus, f. 76 recto-a.

50. - DERIU, Elisabetta, op. cit., p. 536-537.

51. - Guglielmo Lo Monaco (signé) et atelier, Histoire de Ferrante I d'Aragon, porte en bronze, relief. Naples, Castelnuovo, in situ, ca 1474-1477. Voir BARRETO, Joana. «La porte en bronze du Castelnuovo : naissance de la chronique monumentale ». Histoire de l'art, 2004, n54, p. 123-138.

52. - Paolo Uccello (signé), Épisode de la Bataille de San Romano, détrempe sur bois. Florence, Offices, Inventaire $n^{\circ} 52(479)$, datable ca 1435-1436.

53. - DERIU, Elisabetta, op. cit., p. 537.

54. - CENNINI, Cennino. Il Libro dell'arte, ca 1390, chap. LXX « Degli animali non ti chonterò, perché non n'aparai mai nessuna misura; ritra'ne disegnia più che poi del naturale. E proverrai in ciò a buona praticha», éd. FREZZATO, Fabio. Vicence : Neri Pozza, 2003, p. 118. Voir aussi PROCACCI, Ugo. «Disegni per esercitazione degli alievi e disegni preparatori per le opere d'arte nella testimonianza del Cennini ». Studies in Late Medieval and Early Renaissance Painting in honor of Millard Meiss. New York: New York University Press, 1977, p. 352-367; GRAMACCINI, Norberto. "Cennino Cennini e il suo Trattato della pittura », Res Publica Litterarum, Studies in the Classical Tradition, 1987, vol. X, n 19, p. 143-151; BEYER, Andreas, PRINZ, Wolfram. Die Kunst und das Studium der Natur vom 14. zum 16. Jahrhundert. Weinheim : HC, 1987.

55. - Voir AMES-LEWIS, Francis. «Il disegno nella pratica di bottega del Quattrocento ». Dans LUCCO, Mauro. La pittura nel Veneto. Il Quattrocento. Milan : Electa, 1990, vol. II, p. 661 sq. ; ROSSI, Marco. Giovannino de Grassi, la corti e la cattedrale. Cinisello Balsamo: Silvana editoriale, 1995, p. 142-143 ; FILIPPI, Elena. « Les dessins ». Dans PUPPI, Lionello, op. cit., p. 198.

56. - Voir WOODS-MARSDEN, Joanna. « « Draw the irrational animals as often as you can from life » : Cennino Cennini, Giovanni de'Grassi and Antonio Pisanello ». Studi di Storia dell'arte, 1992, vol. 3, p. 67-78.

57. - AUBLET, Henri, Lieutenant-Colonel. Manuel d'hippologie. Paris-Limoges : Lavauzelle, 1975, p. 126. 
58. - ARASSE, Daniel. Le détail. Pour une histoire rapprochée de la peinture. Paris : Flammarion, 1992, rééd., 1996, p. 133.

59. - Ibid., p. 133.

60. - Ibid., p. 133, 186.

61. - EISLER, Colin. The Genius of Jacopo Bellini. The Complete Paintings and Drawings. New York: Harry N. Abram, 1989, p. 78.

62. - Attribué à Giovannino de'Grassi et suiveurs, Taccuino dei disegni. Bergame, Biblioteca Civica, Ms $\Delta$ VII.14, fin du XIV siècle. Voir CADEI, Antonio. Studi di miniatura lombarda, Giovannino de'Grassi, Belbello da Pavia. Rome : Viella, 1984 ; WOODS-MARSDEN, Joanna, op. cit.

63. - S'agissant des hypothèses de reconstitution des carnets de Pisanello, voir CORDELLIER, Dominique, op. cit., p. 457-472.

64. - Unanimement attribué à Jacopo Bellini, Quaderno, sur parchemin. Paris, musée du Louvre, département des Arts graphiques, ca 1460. Voir EISLER, Colin, op. cit.

65. - Unanimement attribué à Jacopo Bellini, Quaderno, sur papier, ca 1440. Londres, British Museum. Voir DEGENHART, Bernhard et SCHMITT, Annegrit. Jacopo Bellini: The Louvre Album of Drawings. New York: Jaca Book, 1984 ; EISLER, Colin, op. cit.

66. - Voir SCHELLER, Robert W. A Survey of Medieval Model Books. Harlem : Erven F. Bohn, 1963, p. 1-37.

67. - Voir WOODS-MARSDEN, Joanna, op. cit., p. 68-69.

68. - Ibid., p. 68-70.

69. - Attribué à Giovannino de'Grassi, Taccuino dei disegni. Bergame, Biblioteca Civica, Ms $\Delta$ VII.14, f. 6 ; attribuée à Pisanello, Étude d'un âne de profil gauche, avec l'esquisse d'un âne et d'un ânier, la reprise de sa croupe et de sa queue. Paris, musée du Louvre, département des Arts graphiques, Inventaire 2458, années 1430. Pour la comparaison des deux dessins, voir CORDELLIER, Dominique, 1996, op. cit., p. 104.

70. - Ibid., p. 104.

71. - Voir notamment PEDRETTI, Carlo. Leonardo da Vinci : Drawings of Horses and other Animals from the Royal Library at Windsor Castle. New York: Johnson Reprint Comp., 1984, p. 38-39. Dans la première édition de ses Vies (1550), Vasari affirme que le traité de Léonard sur l'anatomie équine trouve son origine dans les études pour le monument Sforza et qu'il a été brûlé en 1499, au moment de l'invasion française - ayant également abouti à la destruction du grand modèle en argile du Cavallo. Toutefois, Giampaolo Lomazzo se réfère encore à des études de chevaux de Léonard dans les discours sur les proportions équines intégrés à son Traité de peinture (paru en 1584, livre, chap. XIX, p. 71 et livre II, chap. XII, p. 117) et à l'Idée du temple de la peinture (publiée en 1590, chap. III, p.15) : soit que des notes et/ou des dessins aient été conservés, soit que Lomazzo en ait eu connaissance de manière indirecte.

72. - Madrid, Biblioteca Nacional, Ms II, f. 3.

73. - PEDRETTI, Carlo, op.cit., p. 13, 16.

74. - Voir les notes du manuscrit K3, Paris, Institut de France, f. 109b : citées dans ibid., p. 14 ; RICHTER, Jean-Paul. The Literary Works of Leonardo da Vinci. $2^{\mathrm{e}}$ éd. Oxford : Oxford University Press, 1939, p. 324.

75. - Voir ARASSE, Daniel, op. cit., p. 189.

76. - Léonard de Vinci, Windsor Castle, Royal Library, RL 19070 verso : « Tu prétends qu'il vaut mieux voir pratiquer l'anatomie que regarder mes dessins : tu aurais raison si l'on pouvait voir tous les détails que mes dessins présentent en une seule figure, où, avec tout ton talent, tu ne verras et ne connaîtras que quelques veines. Pour en acquérir une connaissance juste et complète, j'ai disséqué plus de dix cadavres, en détruisant tous les autres éléments, en enlevant jusqu'aux plus petites particules de la chair qui entouraient ces veines, sans autre saignement que celui, tout imperceptible des veines capillaires. Un seul cadavre ne durait pas assez longtemps; il fallait procéder avec plusieurs, par degrés, pour arriver à une connaissance 
complète. Ce que j'ai fait deux fois, pour vérifier les différences ", éd. et trad. françaises : CHASTEL, André. Léonard de Vinci, Traité de la peinture. Paris : Berger-Levrault, 1987, p. 224-225.

77. - Voir KEMP, Martin. «Leonardo's Drawings for « Il Cavallo del duca Francesco Sforza di bronzo » : Program of Research ». Dans AHL, Diane Cole (éd.). Leonardo da Vinci's Sforza Monument Horse: the Art and the Engineering. Bethlehem, Londres, Cranbury: Lehigh University Press, Associated University Presses, 1995, p. 72.

78. - Voir KEMP, Martin, op. cit., p. 65.

79. - Voir n. 19.

80. - Sur ces dessins, voir notamment PEDRETTI, Carlo, op.cit., p. 16 ; FUSCO, Laurie et CORTI, Gino. « Lorenzo de'Medici on the Sforza Monument ». Achademia Leonardi Vinci. Journal of Leonardo Studies and Bibliography of Vinciana, 1992, vol. IV-V, p. 21 ; CLAYTON, Michael. Leonardo da Vinci, a curious vision. Londres : Merrell Holberton, 1996, p. 54.

81. - Unanimement attribuée à Léonard de Vinci, Étude d'un cheval de profil gauche et de ses membres avec mesure, pointe de métal, encre et plume sur papier préparé bleu. Windsor Castle, Royal Library, RL 12319, ca 1493, avec au-dessus du cheval, l'inscription « gianecto.grosso.dj messer Galeazo ".

82. - Unanimement attribuée à Léonard de Vinci, Étude des antérieurs d'un cheval et ses mesures, plume et encre sur pierre rouge sur papier. Windsor Castle, Royal Library, RL 12294, ca 1490, portant l'inscription "Ciciliano dj meser galeazo ». Ce Sicilien est, selon Carlo Pedretti, non seulement le modèle le plus étudié par Léonard, mais aussi celui qui apparaît sur différents dessins du Codex Huygens qui sont des copies réalisées à partir de dessins perdus de Léonard (f. 71, $72,73,80,84,86$ tandis que le f. 77 présente une véritable copie d'un dessin conservé au château de Windsor), ce même Sicilien est encore mentionné dans une brève note du Codex Atlanticus, f. $291 \mathrm{v}$-a. Voir PEDRETTI, Carlo, op. cit., p. 16, 42.

83. - Londres, Victoria and Albert Museum, Codex Forster, f. 88 : «Le cheval florentin noir de Messer Mariolo, un grand cheval, a une encolure fine et une très belle tête / l'étalon blanc appartenant au fauconnier a des hanches fines, il se trouve à Porta Comasina. / Un grand cheval de Cermonino appartenant à Signor Giulio ».

84. - Unanimement attribuée à Léonard de Vinci, Étude pour la statue équestre de Francesco Sforza, pointe d'argent sur papier préparé bleu. Windsor Castle, Royal Library, RL 12357, ca 1484-1489. Unanimement attribuée à Léonard de Vinci, Étude pour la statue équestre de Francesco Sforza, pointe d'argent sur papier préparé bleu. Windsor Castle, Royal Library, RL 12358, ca 1485-1490.

85. - Unanimement attribuées à Léonard de Vinci, Cinq études pour la statue équestre de Gian Giacomo Trivulzio, encre sur papier. Windsor Castle, Royal Library, RL 12353, ca 1506-1511. Unanimement attribuées à Léonard de Vinci, Quatre études pour la statue équestre de Gian Giacomo Trivulzio, encre sur papier. Windsor Castle, Royal Library, RL 12355, ca 1506-1511. Unanimement attribuées à Léonard de Vinci, Trois études pour la statue équestre de Gian Giacomo Trivulzio, pierre noire sur papier préparé. Windsor Castle, Royal Library, RL 12342, ca 1508-1511. Unanimement attribuée à Léonard de Vinci, Étude pour la statue équestre de Gian Giacomo Trivulzio, encre et pierre noire sur papier préparé blanc. Windsor Castle, Royal Library, RL 12343, ca 1508-1511. Unanimement attribuée à Léonard de Vinci, Étude pour la statue équestre de Gian Giacomo Trivulzio, encre et pierre noire sur papier. Windsor Castle, Royal Library, RL 12344 V $^{\circ}$, ca 1508-1511. Unanimement attribuée à Léonard de Vinci, Étude pour la statue équestre de Gian Giacomo Trivulzio, pierre noire sur papier. Windsor Castle, Royal Library, RL 12359, ca 1508-1511. Unanimement attribuée à Léonard de Vinci, Étude pour la statue équestre de Gian Giacomo Trivulzio, encre et craie rouge sur papier. Windsor Castle, Royal Library, RL 12356, ca 1508-1511. Unanimement attribuée à Léonard de Vinci, Étude pour la statue équestre de Gian Giacomo Trivulzio, encre et pierre noire sur papier. Windsor Castle, Royal Library, RL $12356 \mathrm{v}^{\circ}$, ca 1508-1511. Unanimement attribuée à Léonard de Vinci, Étude pour la statue équestre de Gian Giacomo Trivulzio, pierre noire sur papier préparé brun. Windsor Castle, Royal Library, RL 12354, ca 1511. Unanimement attribuées à 
Léonard de Vinci, Cinq études pour la statue équestre de Gian Giacomo Trivulzio, encre et pierre noire sur papier. Windsor Castle, Royal Library, RL 12360, ca 1511.

86. - Windsor Castle, Royal Library, RL 12357 et RL 12358 (voir n. 85).

87. - En particulier Windsor Castle, Royal Library, RL 12342, RL 12359, RL 12356, RL 12356 vº (voir n. 85).

88. - PASTOUREAU, Michel. Figures et couleurs. Études sur la symbolique et la sensibilité médiévales. Paris : Le Léopard d'or, 1996, p. 22.

89. - Voir n. 19.

90. - GAURICUS, Pomponius. De Sculptura, 1504. Éd. et trad. françaises : CHASTEL, André et KLEIN, Robert. Genève : Droz, 1969, p. 204-206.

91. - Parfois attribué à PUCCI, Antonio, ou à BECCARI, Antonio. Sonetto del Cavallo perfetto. Padoue, Biblioteca del Seminario, Ms 59, sonnet 1: voir MARTELLI, Mario. «Il sonetto del cavallo perfetto ». Rinascimento, 1966, vol. 17, nº, p. 57-78 ; DANIELLE, Antonio. La memoria innamorata : indagine e letture petrarchesche. Rome, Padoue : Antenore, 2005, p. 186 ; Id. « Dal Dondi, del Petrarca e di altri. Qualche ipotesi attributive». Dans BRUGNOLO, Furio, VERLATO, Zeno Lorenzo. La cultura volgare padovana nell'età del Petrarca. Monselice : Poligrafo, 2006, p. 388-390.

92. - SEBALD BEHAM, Hans. Dises Buchlein zeyget an und lernet ein mass oder proporcion der Ross. Nüremberg : s.n., 1528.

93. - RUSIO, Lorenzo. Hippiatria sive marescalia. Paris : Chrétien Wechel, 1531.

94. - ALBERTI, Leon Battista. De equo animante. Écrit vers 1445. Editio princeps, Bâle : Michele Stella, 1556. Le cheval vivant. Éd. et trad. françaises : BORIAUD, Jean-Yves. Paris : Les Belles Lettres, 1999.

95. - CLAUT, Paola. «Leon Battista Alberti's De equo animante ». Dans GRAYSON. Studi su Leon Battista Alberti. Florence : Leo S. Olschki, 1998, p. 407-417.

96. - MASTROROSA, Ida. «L'ippiatrica antica e il De equo animante di Leon Battista Alberti ». Dans GATTI, Paolo et DE FINIS, Lia. Dalla tarda latinità agli albori dell'Umanesimo : alle radice della storia europea. Trente : Dipartimento di scienze filologiche e storiche, 1998, p. 192-244.

97. - PLINE L'ANCIEN. Histoire Naturelle, Livre VIII, LXV, 159.

98. - BORIAUD, Jean-Yves. «Préface». Dans ALBERTI, Leon Battista. Le cheval vivant. Paris : Les Belles Lettres, 1999, p. XXXII-XXXIII.

99. - OY-MARRA, Élisabeth. Florentiner Ehrengrabmäler der Frührenaissance. Berlin : Gbr. Mann, 1994, p. 56.

100. - Andrea del Castagno (documenté), Monument funéraire en trompe-l'œil de Niccolò da Tolentino, fresque. Florence, Santa Maria del Fiore, 1456 (documenté).

101. - BERGSTEIN, Mary. "Donatello's Gattamelata and its Humanist Audience ». Renaissance Quaterly, 2002, 55, p. 833-868.

102. - Voir n. 16.

103. - Léonard de Vinci s'est vu confier la commande de la statue équestre de Francesco Sforza à Milan (ca 1485-1494) puis d'une statue équestre pour le monument funéraire de Gian Giacomo Trivulzio (ca 1506-1511), toutes deux à Milan, et peut-être aussi une ultime statue équestre, à l'effigie de François I ${ }^{\text {er }}$ ou d'un autre commanditaire français, dans les dernières années de sa vie passées en France (ca 1517-1518); sur cette hypothétique commande française, très controversée : voir CLAYTON, Michael, op. cit., p. 141-149; ARASSE, Daniel. Léonard de Vinci : le rythme du monde. Rééd. Paris : Hazan, 2011, p. 200.

104. - Unanimement attribuée à Léonard de Vinci, L'Adoration des Mages, détrempe, huile et blanc de plomb sur bois. Florence, galerie des Offices, 1481.

105. - Thèse de PEDRETTI, Carlo, 1984, op. cit., p. 38.

106. - Sur l'analogie homme-cheval dans le travail de Léonard : voir ARASSE, Daniel, 2011, op. cit., p. $67-70$. 
107. - Unanimement attribuée à Léonard de Vinci, Étude d'une tête de cheval de profil droit et d'une tête de cheval de face avec mesures de proportions, mine de plomb, crayon et encre sur papier préparé rose. Windsor Castle, Royal Library, RL 12286, ca 1490.

108. - Unanimement attribuée à Léonard de Vinci avec des traits retracés par Francesco Melzi, Étude de deux antérieurs et de deux postérieurs pliés avec mesures de proportions, crayon et encre sur papier blanc. Windsor Castle, Royal Library, RL 12293, ca 1490. Unanimement attribuée à Léonard de Vinci, Étude d'un antérieur plié du Cheval de Messer Galeazzo avec mesures de proportions, crayon et encre sur papier blanc. Windsor Castle, Royal Library, RL 12294, ca 1490. Unanimement attribuée à Léonard de Vinci, Étude d'un antérieur et postérieur pliés avec mesures de proportions, mine de plomb, crayon et encre sur papier blanc. Windsor Castle, Royal Library, RL 12304 vo ca 1490.

109. - Voir n. 19.

110. - Attribuée à Andrea del Verrocchio, Étude du corps d'un cheval de profil gauche avec mesures des proportions, plume et bistre sur papier blanc. New York, The Metropolitan Museum of Art, Acquisition n¹9.76.3, ca 1483-1488. Attribuée à Andrea del Verrocchio, Étude d'un cheval vu de dos, d'un cheval vu de face et du détail d'un antérieur avec mesures, plume, encre brune et traces de craie noire sur papier. Rome, Istituto Nazionale per la Grafica, FC $127615 \mathrm{v}^{\circ}$, ca 1481.

111. - Unanimement attribuée à Léonard de Vinci, Étude d'un cheval de profil gauche avec mesures de proportions et croupe, encre noire et fusain sur papier blanc. Windsor Castle, Royal Library, RL 12318, ca 1481. Unanimement attribuée à Léonard de Vinci, Étude du Grand Genet de Messer Galeazzo de profil gauche avec mesures de proportions, pointe d'argent sur papier préparé bleu. Windsor Castle, Royal Library, RL 12319, ca 1490.

112. - PANOFSKY, Erwin. The Codex Huygens and Leonardo da Vinci's Art Theory. Londres : Warburg Institute, 1940, p. 106-122; Id. "The History of the Theory of Human Proportions as a Reflection of the History of Styles ». Dans Meaning in the Visual Arts. Rééd. Harmondsworth: Penguin Books, 1970, p. 82-138 ; KEMP, Martin, op. cit., p. 64-78.

113. - Léonard pousse ce fractionnement à l'extrême, jusqu'à calculer des unités d'une valeur d'un centième de la longueur de l'animal : voir LIEDTKE, Walter. The Royal Horse and Rider: painting, sculpture and horsemanship, 1500-1800. New York: Abaris books, 1989, p. 111 ; KEMP, Martin, op. cit. p. 65, 72-73.

114. - PEDRETTI, Carlo, op. cit., p. 41.

115. - ALBERTI, Leon Battista. De pictura. Écrit en 1435. Editio princeps. Bâle : s.n., 1540 ; Id., De statua, écrit vers 1445. Editio princeps: Opuscoli morali. Venise: F. Francesco Senese, 1568, p. 290-305.

116. - PEROCCO, Guido. Les Chevaux de Saint-Marc. Milan : Olivetti, 1981, p. 68.

117. - Jules Romain (Giulio Pippi) et aides (Fermo Ghinsoni, Rinaldo Mantovano, Benedetto Pagni da Pescia, Anselmo Guazzi et Agostino Mozzanega), Les étalons préférés de Federico II Gonzaga, peinture murale, Mantoue, palais du Tè, salon des Chevaux, vers 1528.

118. - Deux documents d'archives datés du 7 février 1529 et du 6 août 1550 mentionnent des représentations peintes (disparues) d'étalons mantouans envoyées à François $\mathrm{I}^{\mathrm{er}}$ par Federico II Gonzaga: Mantoue, Archivio di Stato, Archivio Gonzaga, AG 637 et AG 643 : voir CHATENET, Monique. «Cheval et diplomatie à la cour de France sous François I $^{\text {er }}$ et Henri II : le témoignage des ambassadeurs de Mantoue ». Dans Ead., FRANCHET D'ESPÉREY, Patrice. Les arts de l'équitation dans l'Europe de la Renaissance. Arles : Actes Sud, 2009, p. 56, 65, n. 38.

119. - Anselmo Guazzi, Lucas Fiamingo (documenté), Chambre des chevaux. Mantoue, Palais ducal, peintures murales, disparues, 1536.

120. - DA LUCCA, Silvestro et PADOVANO, Lauro. Codice dei palii gonzageschi. Milan : collection privée des héritiers Alberto Falk. Ancienne collection Biblioteca Giustiniani-Recanati de Venise, vers 1499-1518. 


\section{RÉSUMÉS}

Une des spécificités des portraits équestres italiens des $\mathrm{XIV}^{\mathrm{e}}$ et $\mathrm{XV}^{\mathrm{e}}$ siècles tient dans la double tendance au naturalisme et à l'idéalisation de leurs montures. D'une manière générale, la tension entre individualisation et idéalisation se trouve au cœur de la pensée artistique de la Renaissance, qui fit du portrait son terrain d'élection. Perceptible dans les effigies comme dans toute représentation équestre, y compris les études, via certains modèles équins et détails morphologiques ou physiologiques, la tendance croissante au naturalisme renvoie notamment à la pratique novatrice de la représentation d'après nature. Néanmoins, cette tendance n'empêche pas une certaine idéalisation du cheval, en lien avec un idéal équin théorique et artistique.

Real horses and ideal horses in equestrian portraits of the Trecento and Quattrocento. One of the special characteristics of Italian equestrian portraits during the fourteenth and fifteenth centuries, where the depiction of the mounted horse is concerned, is the dual tendency towards naturalism on the one hand and towards idealisation on the other. More broadly speaking, this tension between individualisation and idealisation is at the heart of the artistic thinking of the Renaissance period, which was particularly keen on the portrait. The trend towards naturalism, which can be perceived in effigies, in other equestrian representations and in morphological or physiological studies and models, refers to the novel practice of painting after nature. But this does not eliminate a certain idealisation of the horse, deriving from a theoretical and artistic ideal.

\section{INDEX}

Mots-clés : naturalisme, idéalisation, portrait équestre, représentation équestre, cheval, morphologie, mouvement, Leon Battista Alberti, Pisanello, Léonard de Vinci

Keywords : naturalism, equestrain portrait, equestrian representation, horse, morphology, movement

\section{AUTEUR}

\section{ARMELLE FÉMELAT}

Chercheur associé au CESR (Centre d'Etudes Supérieures de la Renaissance)

armelle.femelat@gmail.com 